Article

\title{
Thermodynamic and Economic Analysis of Trigeneration System Comprising a Hierarchical Gas-Gas Engine for Production of Electricity, Heat and Cold
}

\author{
Ryszard Bartnik, Zbigniew Buryn $₫$, Anna Hnydiuk-Stefan *๑) Waldemar Skomudek and \\ Aleksandra Otawa \\ Department of Power Management, Faculty of Production Engineering and Logistics, Opole University of \\ Technology, 76 Prószkowska str., 45-768 Opole, Poland; r.bartnik@po.edu.pl (R.B.); z.buryn@po.edu.pl (Z.B.); \\ w.skomudek@po.edu.pl (W.S.); a.otawa@po.edu.pl (A.O.) \\ * Correspondence: a.hnydiuk-stefan@po.edu.pl; Tel.: +48-77-449-8730
}

Received: 2 February 2020; Accepted: 19 February 2020; Published: 24 February 2020

check for updates

\begin{abstract}
This paper presents the results of analysis of energy and economic efficiency of the hierarchical gas-gas engine, with a note that a trigeneration system was analyzed, in which the production of electricity, heat and cold are combined. This solution significantly increases the energy efficiency of the gas and gas system compared to a system without cold production. The analysis includes a system comprising a compressor chiller which is driven by an electric motor in the system, as well as a system applying the mechanical work that is carried out via a rotating shaft of rotor-based machines, i.e., a gas turbine and a turboexpander. The comfort of the regulation of the refrigerating power rather promotes the use of a solution including an electric motor. Analysis contains also a schematic diagram of the system with a absorption chiller, which is driven by low-temperature enthalpy of exhaust gases extracted from a hierarchical gas-gas engine. Application of turboexpander with heat regeneration in the trigeneration system is also analyzed. Based on the multi-variant economic and thermodynamic calculations, the most favorable system variant was determined using, among others, the specific cost of cold production.
\end{abstract}

Keywords: trigeneration; electricity; heat; cold; efficiency of the gas-gas system; heat regeneration in the turboexpander; heat regeneration in the gas turbine

\section{Introduction}

In view of the growing demand for electricity, it is important to use effective energy systems. Separate electricity and heat generation is energy inefficient because it requires more fuel to be consumed than when produced simultaneously. An even better solution in terms of fuel saving is the simultaneous production of electricity, heat and cold, which is the subject of this article.

The energy and economic efficiency of two alternatives involving an innovative, hierarchical two-cycle gas-gas engine operation were analyzed in [1]. In this design, two Joule circulations were combined and implemented, i.e., a high-temperature Joule cycle of a gas turbine and a low-temperature Joule turboexpander cycle. The coupling of the circulations takes place as a result of the use of a flue gas-air system in the air heater $\mathrm{N}$ - Figures $1-3$. In one alternative, only electricity is produced, in the other, combined electricity and heat generation is conducted. The energy efficiency of both designs is lower than the efficiency of a hierarchical gas-steam engine with two circulation systems, in which the Joule cycle of a gas turbine operating in a high temperature range is coupled with the circulation of a Clausius-Rankine steam turbine, operating in the low temperature range. As a result of coupling 
the Joule and Clausius-Rankine through a gas-steam-water system in a heat recovery steam generator, which uses a low-temperature enthalpy of exhaust gases extracted from a gas turbine to produce steam to feed the steam turbine [2]. The higher efficiency of a gas-steam engine is determined by the steam condensing isotherm (which of course also forms an isobar), during which heat is released into the environment from the Clausius-Rankine cycle. This isotherm nearly overlaps with the isotherm of the most thermodynamically ideal, theoretical circuit of the Carnot engine characterized by the highest thermal efficiency that mechanical engines can achieve [3]. However, the circulating medium in the isobaric transformation of the "lower" Joule cycle in a hierarchical gas-gas engine, during which heat is released from the engine to the environment, has a significantly higher temperature compared to the entropy-averaged temperature $T_{a m b}[2,4]$. As a consequence, the ratio of the power of the turboexpander to the one of the gas turbine $N^{T E} / N^{G T}$ in a gas-gas system for the case of production of only electricity in both systems is even more than three times smaller than the ratio of steam turbine power to gas turbine power $N^{S T} / N^{G T}$ in a gas-steam system. In the case of operation of both systems in a combined cycle, the ratio $N^{T E} / N^{G T}$ is about two times smaller than $N^{S T} / N^{G T}$ [1]. Higher electricity production in the gas-steam system results from the built-in heat recovery steam generator, which makes it possible to apply the low-temperature enthalpy of exhaust gases from the turbine throughout the year for steam production applied to feed the steam turbine [2]. The temperature of the gas extracted from the heat-recovery steam generator and released the environment, both for the combined cycle and the purely condensing operation of the gas-steam system, is low all year, as it is in the range from 70 to $90^{\circ} \mathrm{C}$, whereas in the gas-gas system the temperature of the exhaust gas to the environment is relatively high. In the case of a system applied for the production of only electricity (this system does not contain the heat exchanger (HE) in Figures 1 and 2), the temperature of the exhaust gas fed into the stack is in the range of about 180 to $250{ }^{\circ} \mathrm{C}$ (this temperature depends on the temperature of the gas entering the gas turbine [1]), and in the case of combined operation in the heating season in the range of about 130 to $160^{\circ} \mathrm{C}$, and in the non-heating season in the range of about 170 to $240{ }^{\circ} \mathrm{C}$ [1]. Of course, these are then the flue gas temperatures behind the heat exchanger (HE), Figures 1 and 2.

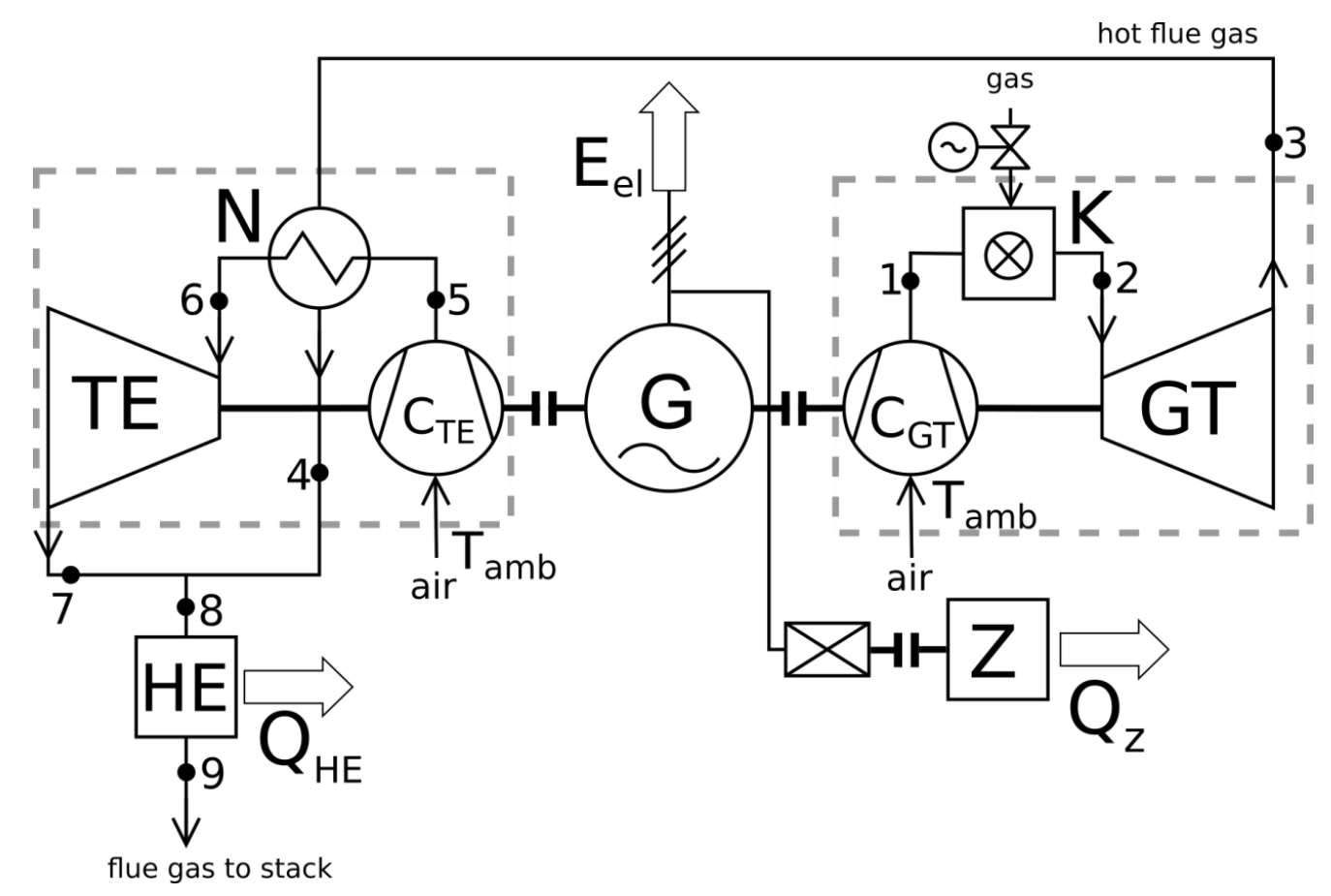

Figure 1. Schematic diagram of a system including compressor chiller driven by an electric motor. 


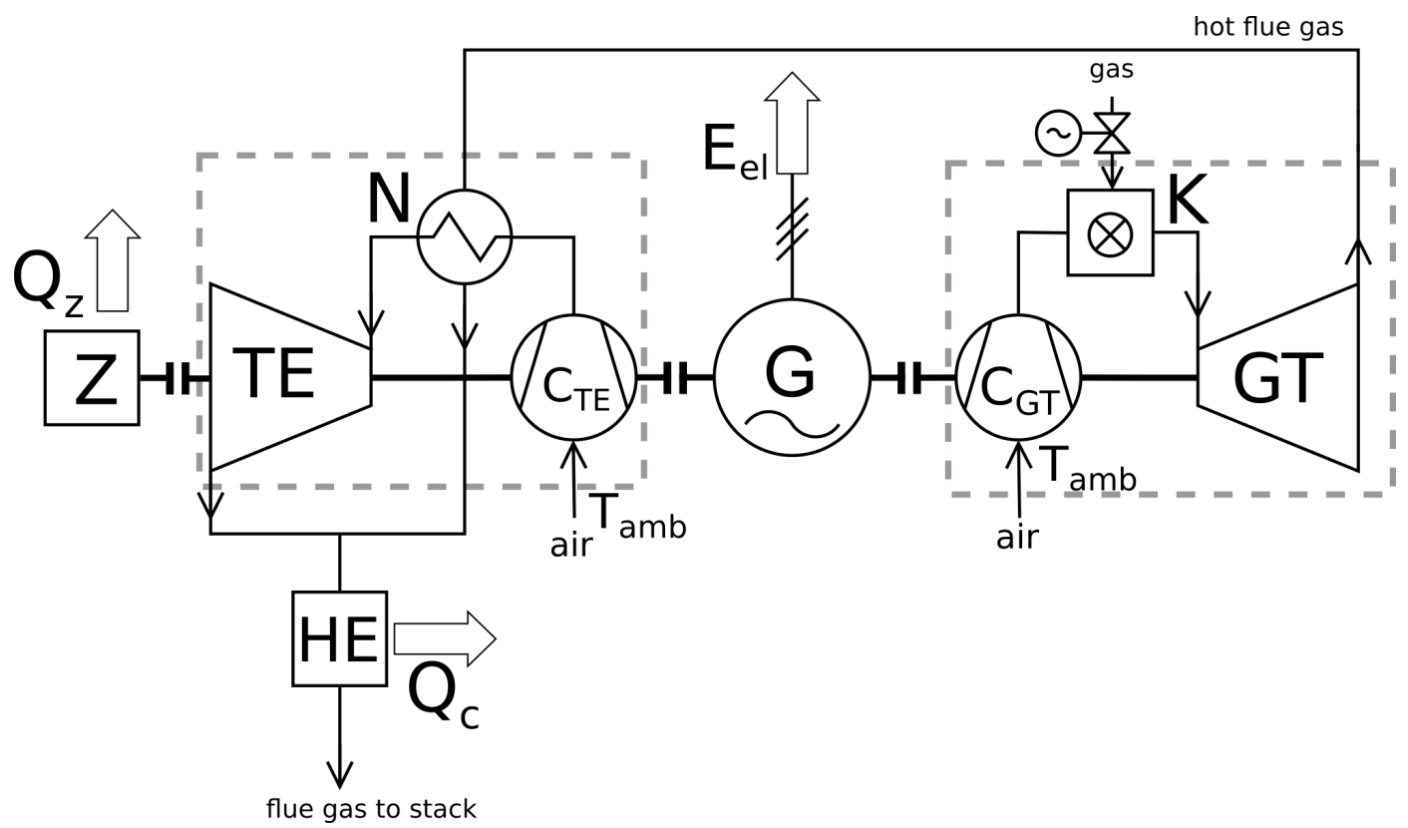

Figure 2. Schematic diagram of a system including compressor chiller located on a common shaft with a gas turbine and turboexpander.

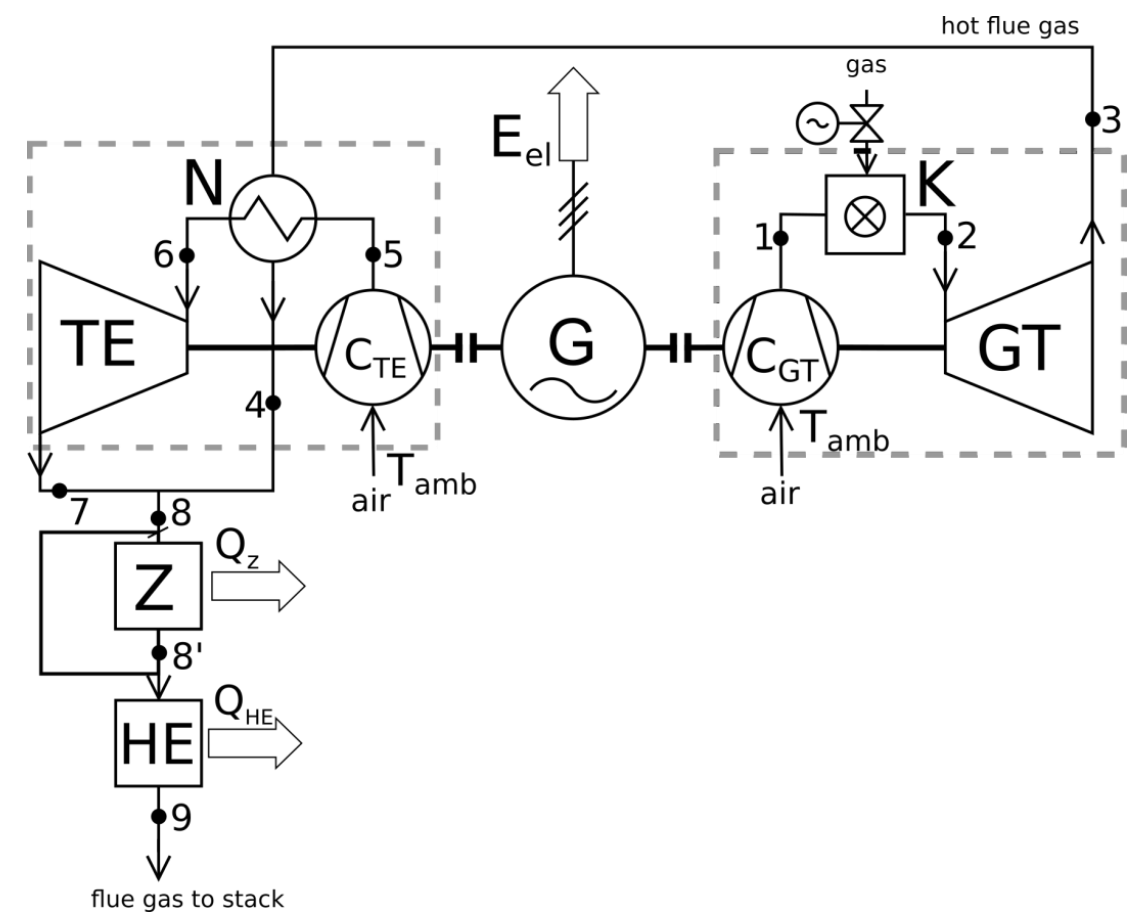

Figure 3. Schematic diagram of a trigeneration hierarchical gas-gas system with a gas turbine and turboexpander in a single-shaft configuration designed for the production of electricity, heat and cold in an absorption chiller ( $\mathrm{G}$ - electric generator, $\mathrm{K}$ - combustion chamber of the gas turbine, $\mathrm{N}$-air preheater; preheater is a device that combines the circulation of the gas turbine and that of the turboexpander, $C_{T E}$ - low-pressure compressor, $C_{G T}$-high-pressure compressor of the gas turbine, GT-turbine section of the gas turbine, TE-turboexpander, HE-heat exchanger, Z-absorption chiller, $1 \div 9$ - points along the system).

However, what is important is that the economic effectiveness of the hierarchical gas-gas engine operating in the combined cycle is greater than the effectiveness of the hierarchical gas-steam system operating according to a combined cycle, and the economic effectiveness of gas-gas and gas-steam 
systems applied for the production of only electricity are comparable, despite lower electricity production in the gas-gas system [2]. The better economic effectiveness of gas-gas engines is associated with the significantly lower specific expenditure associated with the investment in the system (calculated per unit of the installed electrical capacity). This ratio is around $45 \%$ of the specific expenditure needed on gas-steam systems [2,4].

In [5] the processes of power, heating and cooling production, called CHP (combined heat and power) and CCHP (combined cooling heat and power) systems are elaborated. The overall structure of the units and likewise the inputs and outputs of them for a better understanding and comparison are the main features of this paper. Also, consumed fuels, applied oxygen carriers, the main conditions in chemical looping units, the chemical reactions and the resulting efficiencies are considered and discussed. A novel cascade trigeneration system driven by a biofuel production source (BPS) from the textile wastewater is introduced in [6] and modeled energetically and economically. The CCHP system comprises a Brayton top power cycle (BC) and three bottoming sub-systems, including the Rankine power cycle (RC), modified Kalina/vapor-compression refrigeration system (KVC34), and water-heating unit (WHU). The feasibility demonstration and parametrical optimization of the proposed system were conducted from thermal, technical, and economic points of view.

The study [7] investigates the feasibility of utilizing waste heat from industrial processes for the purpose of generating chilled water and hot water to be used for space conditioning and/or process heating applications. Some potential strategies to enhance heat source temperature are pointed out in this study, along with the anticipated outcomes of such temperature increases. Also, systems specifically tailored to chilling-only or water heating-only applications may hold potential for improved system performance.

The results of a thermodynamic-economic-environmental analysis of conventional and integrated solar gas turbine trigeneration power plants based on parabolic trough collectors are presented in [8]. The hourly and yearly performance of the plants considered with different gas turbine and solar field sizes have been examined and presented. In addition, a conceptual procedure to identify the optimal solar integration configuration has been developed and presented.

Paper [9] discusses the possibilities of integrating the adsorption aggregate with a combined cycle gas turbine and its impact on the operation of all devices. The results obtained confirm that the adsorption aggregate, using a low grade of thermal energy, does not affect the operation of the gas and steam cycle, and allows the production of electricity at a constant level.

This manuscript [10] presents a thermo-economic analysis for a trigeneration system integrated by an absorption refrigeration chiller, a gas microturbine, and the heat recovery steam generation subsystem. The effect of the compressor inlet air temperature on the thermo-economic performance of the trigeneration system was studied and analyzed in detail based on a validated model. The critical operating conditions for which the trigeneration system presents the greatest exergy destruction, producing an increase in the costs associated with loss of exergy, relative costs, and operation and maintenance costs were determined.

The paper [11] proposes a novel methodology for developing an insight-based numerical pinch analysis technique to simultaneously target the minimum cooling, heating and power requirements for a total site energy system. It enables the design of an integrated centralised trigeneration system involving several industrial sites generating the same utilities.

The paper [12] presents a case study and simulation calculations of the system composed of molten hydroxide electrolyte (MH-DCFC) that generates electricity, and runs heat exchangers and an absorption chiller, integrated with the fuel cell to generate heating and cooling for improving the efficiency of the whole system. The maximum heat and cool streams that can be obtained during the operation of the cell were also evaluated. The results obtained in the study can be helpful in the design of autonomous buildings equipped with direct carbon fuel cells as sources of electricity integrated with the systems of heat and cool generation. 
The objective of the paper [13] is to optimize the daily plant operation of an existing trigeneration plant based on cogeneration engines and to study the optimal cooling load-sharing between different types of absorption chillers using a mixed integer linear programming (MILP) model.

The scheduling of both absorption cycle and vapour compression cycle chillers in trigeneration plants is investigated in [14].

In [15] a thermodynamic study was conducted on the energetic and exergy performance of a new configuration of a liquid chemical looping gasification (LCLG) plant integrated with a power block to assess the overall performance of the system including exergy partitioned in syngas and first law efficiency (FLE).

This paper presents the results of analysis of energy and economic efficiency of the above hierarchical gas-gas engine, with a note that a trigeneration system was analyzed, in which the production of electricity, heat and cold are combined, Figures 1-3. This solution significantly increases the energy efficiency of the gas and gas system compared to a system without cold production. Development of trigeneration schemes is a promising solution to energy and environmental problems by increasing energy efficiency and minimizing energy losses [16-19].

In the system presented in Figure 1, a compressor chiller is installed, which is driven by an electric motor, in the system in Figure 2, applying the mechanical work that is carried out via a rotating shaft of rotor-based machines, i.e., a gas turbine and a turboexpander. The comfort of the regulation of the refrigerating power rather promotes the use of a solution including an electric motor.

Figure 3 contains a schematic diagram of the system with a absorption chiller, which is driven by low-temperature enthalpy of exhaust gases extracted from a hierarchical gas-gas engine.

As a result, the revenue from the sale of electricity produced in the system is not depleted compared to the systems presented in Figures 1 and 2. For the drive of compressor chillers, as already noted above, we apply the electricity (mechanical work, Figure 2), which is generated in the system, which decreases the revenue from its sale. On the other hand, one of the advantages of compressor chillers is associated with their high cooling power, and therefore considerable value of sales of cold, whereas in the system comprising an absorption chiller, its production is relatively small. This is due to the limited range of temperature of the flue gas applied to drive the chiller. The production of heat is also relatively low due to the use of flue gas enthalpy not only for its production, but also, as already noted above, for the production of cold, Figure 3.

In the analyzed trigeneration system, as in the systems in [1], it is beneficial to install a turboexpander and a low-pressure compressor in a single installation and on a common shaft, which is presented by symbols in Figures 1-3 using dashed squares, identically, as is the case for the turbine part and gas turbine compressor. Such a solution reduces the necessary investment in a gas-gas engine.

On the basis of the analysis of the performance of the hierarchical, trigeneration gas-gas system, it is also reasonable to find the answer to the question: to what extent does the use of heat regeneration in the turboexpander-Figure 4-as well as in the gas turbine [1] increase its capacity, i.e., electric, thermal and cooling power. A significant increase in any of them would be converted into greater economic feasibility of their operation. We can note here that the use of regeneration does not increase the stream of the chemical energy $\dot{E}_{c h}$ of gas combustion in the gas turbine and, therefore, does not increase the cost of fuel. In a hierarchical system, the fuel is supplied only to the circulation that performs operations in the highest temperature ranges. For each of the subsequent circulations in the hierarchical system that operate in the lower temperature ranges, the heat that is routed to drive them comes from heat derived from the circulation in the hierarchy located just above them. 


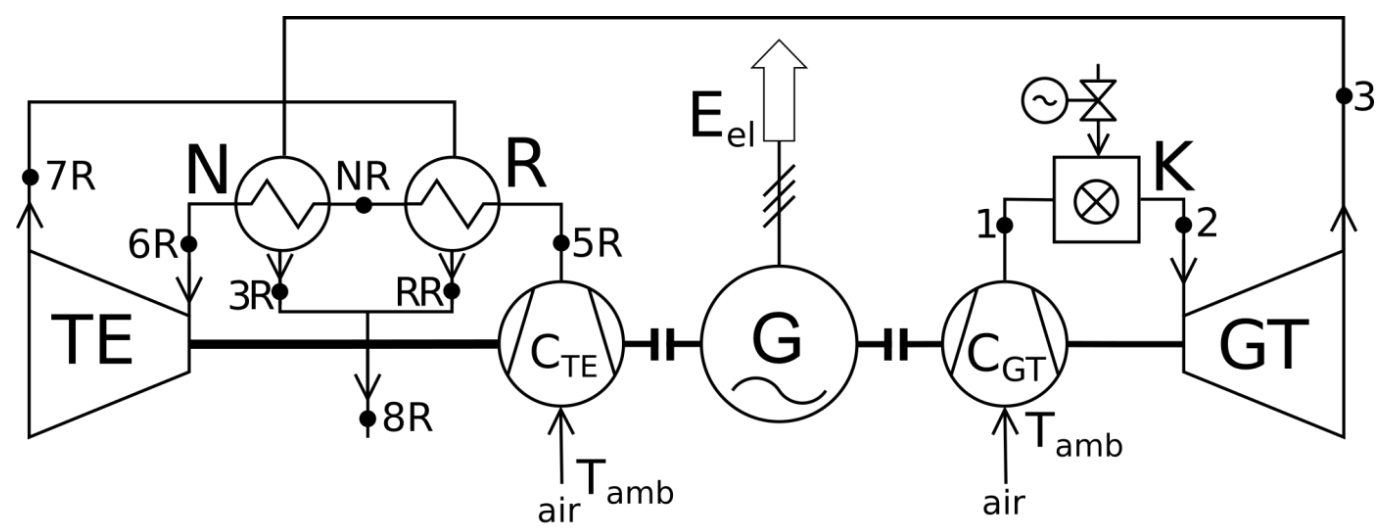

Figure 4. Schematic diagram of a hierarchical gas-gas engine with turboexpander operating with heat regeneration ( $\mathrm{R}$-regenerative heat exchanger).

\section{Thermodynamic and Economic Analysis of the System for Combined Production of Electricity,} Heat and Cold

Thermodynamic analysis provides a way to search for opportunities to improve technological processes and design solutions of machines and equipment. However, in a market economy the economic criterion and the desire to gain a profit and its maximization ultimately determines the feasibility of using specific technical solutions, and economic effectiveness analysis forms the basis for taking decisions regarding investments. The economic criterion is superior to the technical criterion. However, it should be strongly emphasized that economic analysis is only possible after previous thermodynamic analysis. Its results offer the input values for the subsequent economic analysis.

\subsection{Analysis of Exergy of the Hierarchy System of Gas-Gas Engine Driving the Compressor Chiller}

The balance of exergy of the hierarchy system of gas-gas engine driving the compressor chiller has been presented below - Figure 5 .

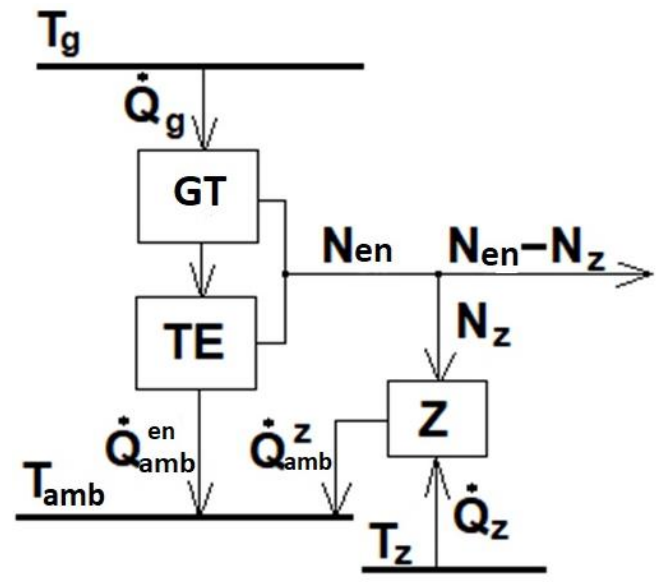

Figure 5. Schematic diagram of the hierarchy system of gas-gas engine driving the compressor chiller $\mathrm{Z}$ for illustration of the exergy balance.

Exergy balance of the system (engine + chiller) for its preset operation can be reflected by the following relationship:

$$
0=\sum_{m=2} \Delta \dot{B}_{S}+N_{e n}-N_{Z}+\delta \dot{B}
$$

where:

$\Delta \dot{B}_{S}$-increase of exergy of an external source of heat delivered to the system, 
$N_{e n}$-power of the hierarchic gas-gas engine; the engine power is the sum of gas turbine and turboexpander power, $N_{e n}=N^{G T}+N^{T E}$,

$\mathrm{N}_{Z}$-chiller driving power.

The increase of exergy of external sources of heat delivered to the system can be reflected by the following relationship:

$$
\sum_{m=2} \Delta \dot{B}_{S}=\Delta \dot{B}_{S}^{T_{g}}+\Delta \dot{B}_{S}^{T_{Z}}=-\dot{Q}_{g} \frac{T_{g}-T_{a m b}}{T_{g}}-\dot{Q}_{Z} \frac{T_{Z}-T_{a m b}}{T_{Z}} .
$$

As follows from Equation (2), exergy of heat source with a temperature of $T_{g}$, from which a stream of powering heat $\dot{Q}_{g}$ is collected, decreases, where the exergy drop $\Delta \dot{B}_{S}^{T_{g}}$ is lower than the amount of heat $\dot{Q}_{g}$. In case of the chiller, exergy of cooling chamber with temperature of $T_{Z}$, from which a stream of heat is collected $\dot{Q}_{Z}$, increases, while directions of heat streams $\dot{Q}_{Z}$ and exergy $\Delta \dot{B}_{S}^{T_{Z}}$ are opposite in this case.

Loss of exergy in the system can be reflected by the following relationship:

$$
\delta \dot{B}=T_{a m b} \sum_{l=3} \Delta \dot{S}_{S}=T_{a m b}\left(\frac{\dot{Q}_{a m b}^{e n}+\dot{Q}_{a m b}^{Z}}{T_{a m b}}-\frac{\dot{Q}_{g}}{T_{g}}-\frac{\dot{Q}_{Z}}{T_{Z}}\right),
$$

where the heat stream $\dot{Q}_{g}$ is identical to the stream of chemical energy of fuel burned in the combustion chamber of the gas turbine, $\dot{Q}_{g} \equiv \dot{E}_{c h}$. The fuel combustion temperature is $T_{g}$.

The power derived from the system can, for instance, be determined by use of exergy balance formula (1), by substituting Equations (2) and (3):

$$
N_{e n}-N_{z}=\dot{Q}_{g} \frac{T_{g}-T_{a m b}}{T_{g}}+\dot{Q}_{Z} \frac{T_{Z}-T_{a m b}}{T_{Z}}-\delta \dot{B}=\dot{Q}_{g}+\dot{Q}_{Z}-\dot{Q}_{a m b}^{e n}-\dot{Q}_{a m b}^{Z}
$$

Equation (4), its final form, can be also calculated directly from the system energy balance. It is demonstrated by the left and right side of the equation.

Heat streams transmitted to the environment from the hierarchic two-cycle engine $\dot{Q}_{a m b}^{e n}$ and the most efficient one-cycle chiller with best thermodynamic features $\dot{Q}_{a m b}^{Z}$ are reflected by the following equations, respectively:

$$
\begin{gathered}
\dot{Q}_{a m b}^{e n}=\dot{Q}_{g}\left(1-\eta_{e n}\right)=\dot{Q}_{g}\left[1-\left(\eta_{G T}+\eta_{T E}-\eta_{G T} \eta_{T E}\right)\right], \\
\dot{Q}_{a m b}^{Z}=\dot{Q}_{Z}\left(\frac{1}{\varepsilon}+1\right),
\end{gathered}
$$

where efficiency of the chiller is reflected by the following equation:

$$
\varepsilon=\frac{\dot{Q}_{Z}}{N_{Z}}
$$

A detailed analysis of thermodynamics and efficiency of trigeneration system with hierarchic gas-gas engine for cogeneration of electricity, heat and cold has been presented below.

\subsection{Analysis of System Comprising Compressor Chiller}

The economic analysis of trigeneration system-Figures 1-3-accounts for the input quantities: gas turbine power $N^{G T}$, turboexpander power $N^{T E}$, thermal power $\dot{Q}_{c}$ of the heat exchanger, cooling power $\dot{Q}_{z}$ of either the compressor or absorption chiller, as well as the stream of the chemical energy of 
the gas $\dot{E}_{c h}$ burned in the gas turbine. We can emphasize at this point that the power of the gas turbine $N^{G T}$ determines the remaining quantities: $N^{T E}=f\left(N^{G T}\right), \dot{Q}_{c}=f\left(N^{G T}\right), \dot{Q}_{z}=f\left(N^{G T}\right)$ and obviously decides on $\dot{E}_{c h}=f\left(N^{G T}\right)$. Hence, the power of the gas turbine $N^{G T}$ and the temperatures of the flue gas $T_{2}$ fed into it determine the capacity of the examined trigeneration system. Another important matter is associated with the development of a mathematical model that can provide the account of the economic profitability of the trigeneration system in such a way that it can be employed to generalize the results of the study, so that the resulting calculations can be applied with regard to any power of the gas turbine, and thus apply to any alternative of the power of the trigeneration system. For this purpose, only dimensionless quantities were applied in the model: $N^{T E} / N^{G T}, \dot{Q}_{c} / N^{G T}, \dot{Q}_{z} / N^{G T}$, $\dot{E}_{c h} / N^{G T}$.This is due to the fact that only dimensionless approach can be applied to generalize the considerations, i.e., it offers the assessment of the economic effectiveness of the performance of the trigeneration system with any electrical, heat and cooling power.

As a consequence, the comprehensive figures are employed in this study, in other words they can be applied to any power of the investigated system. These powers, as we already noted and we can emphasize once again, are determined only by the power of the gas turbine $N^{G T}$ and the temperature $T_{2}$ of the gases extracted from the combustion chamber $\mathrm{K}$ of the gas turbine. This temperature $T_{2}$ forms the basic characteristic of a turbine and is always given as part of the manufacturer's data. As a last resort, to know the specific cost of cold generation in the trigeneration systems discussed in this paper, it is sufficient to know the temperature $T_{2}$ of the gas extracted to feed to the gas turbine as well as the current prices of gas, electricity and heat- Figures 7-12, Figures 15-20.

The fundamental quantities that determine the economic feasibility of gas-gas trigeneration systems include the energy efficiency of a gas turbine $\eta_{G T}$ and turbo-expander $\eta_{T E}$ operating according to Joule's cycles. They are determined using energy balances.

On the basis of the Joule's circulation of the gas turbine, we obtain (Equations (8)-(18) [20]):

$$
\begin{gathered}
\eta_{G T}=\frac{N^{G T}}{\dot{E}_{c h}}=\frac{N_{i \exp }-N_{i C}}{\dot{E}_{c h}}=\frac{\eta_{m}\left(T_{2}-T_{3}\right)-\frac{1}{\eta_{m}}\left(T_{1}-T_{a m b}\right)}{T_{2}-T_{1}}= \\
=\frac{\eta_{m}^{2} \eta_{i}^{C} \eta_{i}^{G T} T_{2}\left(1-\frac{1}{z_{G T}}\right)-T_{a m b}\left(z_{G T}-1\right)}{\eta_{m} \eta_{i}^{C}\left(T_{2}-T_{a m b}\right)-\eta_{m} T_{a m b}\left(z_{G T}-1\right)} \rightarrow \max
\end{gathered}
$$

The efficiency $\eta_{G T}$ is the greater along with the bigger temperature $T_{2}$. This temperature is limited only by the heat resistance ability of the blading system in a turbine.

The final form of Equation (1) can be derived after we substitute the irreversible adiabates amb-1 and 2-3 into it-Figure 6:

$$
\begin{gathered}
T_{1}=T_{a m b}+\frac{1}{\eta_{i}^{C}}\left(T_{1 s}-T_{a m b}\right), \\
T_{3}=T_{2}-\eta_{i}^{G T}\left(T_{2}-T_{3 s}\right),
\end{gathered}
$$

where:

$$
z_{G T}=\frac{T_{1 s}}{T_{a m b}}=\frac{T_{2}}{T_{3 s}}=\left(\frac{p_{1}}{p_{a m b}}\right)^{\frac{\kappa-1}{\kappa}}
$$

From the formula (8), we can derived the optimum ratio of the pressure $z_{G T}^{o p t}$, for which the circulation assumes its maximum efficiency $\eta_{G T}^{\max }$, hence, when its maximum power is achieved (an identical procedure is followed when we need to establish the maximum efficiency $\eta_{T E}^{\max }$ of the turboexpander). The ratio $z_{G T}^{o p t}$ forms the function of the temperatures $T_{a m b}, T_{2}$ and the mechanical efficiency $\eta_{m}$ of the compressor and turbine (in the analysis we assumed that the mechanical efficiency 
of the compressor and turbine are the same) and the internal efficiency of the compressor $\eta_{i}^{C}$ and turbine $\eta_{i}^{G T}$ :

$$
z_{G T}^{o p t}\left(T_{a m b}, T_{2}, \eta_{m}, \eta_{i}^{C}, \eta_{i}^{G T}\right)=\left(\frac{p_{1}}{p_{a m b}}\right)_{o p t}^{\frac{\kappa-1}{\kappa}}
$$

where:

$\kappa$ - isentropic exponent of the circulating medium (in the calculations it was assumed that $\kappa=1.4$ ),

$p_{1}=p_{2}, p_{a m b}$ - pressure of the circulating medium accompanying the heat absorption and emission (in the calculations we assumed that $p_{a m b}=0.1 \mathrm{MPa}$ ).

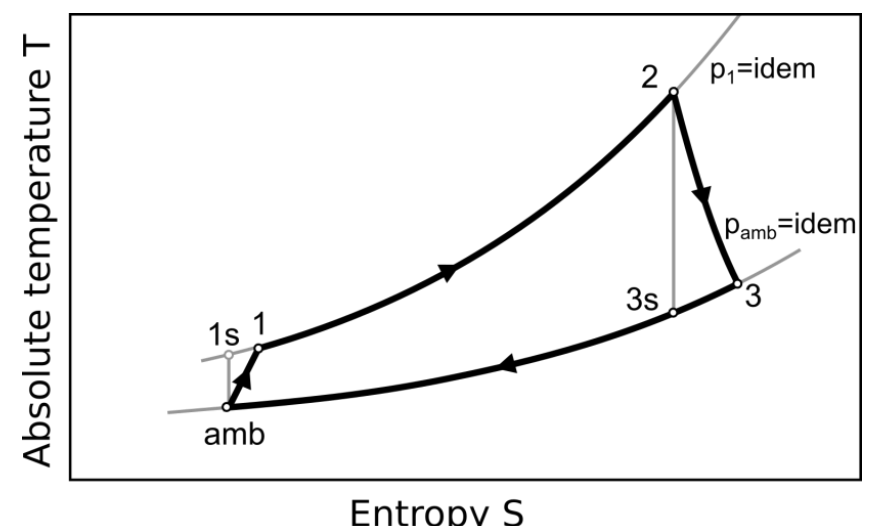

Figure 6. Joule's cycle in a gas turbine.

The thermodynamic calculations assumed an ambient temperature of $T_{a m b}=288 \mathrm{~K}$ and the mechanical efficiency of the compressor and gas turbine are the same and equal to $\eta_{m}^{C}=\eta_{m}^{G T}=\eta_{m}=$ 0.97 , and the internal efficiencies are equal to $\eta_{i}^{G T}=0.87, \eta_{i}^{C}=0.85$.

The optimum value of $z_{G T}^{\text {opt }}$ results from the condition that:

$$
\frac{d \eta_{G T}}{d z_{G T}}=0
$$

Following the differentiation of Equation (8) and application of the condition (13), we obtain:

$$
(b c-a d)\left(z_{G T}^{o p t}\right)^{2}-2 b c z_{G T}^{o p t}+b(c+d)=0,
$$

Hence

$$
z_{G T}^{o p t}=\frac{b c-\sqrt{b d(a d+a c-b c)}}{(b c-a d)}
$$

where:

$$
a=T_{a m b}, b=\eta_{m}^{2} \eta_{i}^{C} \eta_{i}^{G T} T_{2}, c=\eta_{m} T_{a m b}, d=\eta_{m} \eta_{i}^{C}\left(T_{2}-T_{a m b}\right)
$$

The square root of Equation (14) is unfeasible, since the temperature $T_{1}$ would then be greater than temperature $T_{2}$.

The maximum value of $\eta_{G T}^{\max }$ is derived from Equation (8) after the value $z_{G T}^{o p t}$ is applied to replace $z_{G T}$. As a consequence, for $\eta_{G T}^{\max }$ and a given value of $\dot{E}_{c h}$ we can derive the maximum power of the gas turbine that is applied in the economic analysis. In the manufactured gas turbines, $z_{G T}$ assumes the value of $z_{G T}^{\text {opt }}$. 
The temperatures of the circulating medium behind the compressor $T_{1}$ and behind the turbine $T_{3}$ are derived on the basis of irreversible adiabatic processes:

$$
\begin{aligned}
& T_{1}=T_{a m b}\left[1+\frac{1}{\eta_{i}^{C}}\left(z_{G T}^{o p t}-1\right)\right], \\
& T_{3}=T_{2}\left[1-\eta_{i}^{G T}\left(1-\frac{1}{z_{G T}^{o p t}}\right)\right] .
\end{aligned}
$$

As noted already, an identical procedure is applied to obtain $z_{T E}^{\text {opt }}$ and $\eta_{T E}^{\max }$ with a note that in the place of the temperatures $T_{1}, T_{2}$ and $T_{3}$ in Formulae (8), (12), (16), (17) and (18), we need to substitute temperatures: $T_{5}, T_{6}$ and $T_{7}$, and the pressure $p_{5}$ is applied to replace the value of $p_{1}$ in Formula (12). The maximum capacities expressed by $N^{G T}, N^{T E}$ that are derived on the basis of values $\eta_{G T}^{\max }, \eta_{T E}^{\max }$ form the input quantities for the economic calculations (Formula (19)). The results of thermodynamic calculations for the gas turbine set and turboexpander are presented in Figures 24-26.

As indicated above, the capacities $N^{G T}, N^{T E}$ derived from thermodynamic analysis form the input for the further economic analysis. Its purpose involves the determination of the specific cost of the production of cold. It is calculated using the formula for the discounted value of NPV that is obtained throughout $T$ years of operation of the trigeneration gas and gas system applied for combined production of electricity, heat and cold [21-23]:

$$
\begin{aligned}
& N P V=\left\{\left[\left(N^{G T}+N^{T E}\right) \eta_{G} \tau_{R}-N_{z} \tau_{z}\right]\left(1-\varepsilon_{e l}\right) \frac{e_{e l}^{t=0}}{a_{e l}-r}\left[\mathrm{e}^{\left(a_{e l}-r\right) T}-1\right]+Q_{R c} \frac{e_{c}^{t=0}}{a_{c}-r}\left[\mathrm{e}^{\left(a_{c}-r\right) T}-1\right]+\right. \\
& +Q_{R z} \frac{e_{z}^{t=0}}{a_{z}-r}\left[\mathrm{e}^{\left(a_{z}-r\right) T}-1\right]-\dot{E}_{c h} \tau_{R}\left\{\frac{e_{\text {fuel }}^{t=0}}{a_{\text {fuel }}-r}\left[\mathrm{e}^{\left(a_{\text {fuel }}-r\right) T}-1\right]+\right. \\
& +\frac{\rho_{\mathrm{CO}_{2}} t_{\mathrm{CO}}^{t=0}}{a_{\mathrm{CO}_{2}-r}}\left[\mathrm{e}^{\left(a_{\mathrm{CO}_{2}}-r\right) T}-1\right]+\frac{\rho_{\mathrm{CO}} p_{\mathrm{CO}}^{t=0}}{a_{\mathrm{CO}}-r}\left[\mathrm{e}^{\left(a_{\mathrm{CO}}-r\right) T}-1\right]+ \\
& +\frac{\rho_{\mathrm{NO}_{X}} p_{\mathrm{NO}}^{t=0}}{a_{\mathrm{NO}_{X}}-r}\left[\mathrm{e}^{\left(a_{\mathrm{NO}_{X}}-r\right) T}-1\right]+\frac{\rho_{\mathrm{SO}_{2}} p_{\mathrm{SO}_{2}}^{t=0}}{a_{\mathrm{SO}_{2}}-r}\left[\mathrm{e}^{\left(a_{\mathrm{SO}_{2}}-r\right) T}-1\right]+ \\
& \left.+\frac{\rho_{\text {dust }} p_{\text {dust }}^{t=0}}{a_{\text {dust }}-r}\left[\mathrm{e}^{\left(a_{\text {dust }}-r\right) T}-1\right]+\frac{\rho_{\mathrm{CO}_{2}} e_{\mathrm{CO}}^{t=0}}{b_{\mathrm{CO}_{2}}-r}\left[\mathrm{e}^{\left(b_{\mathrm{CO}_{2}}-r\right) T}-1\right]\right\}+ \\
& \left.-\left(1+x_{\text {sal }, t, i n s}\right)\left(J_{G-G}+J_{z}\right)\left(1-\mathrm{e}^{-r T}\right) \frac{\delta_{\text {serv }}}{r}-z\left(J_{G-G}+J_{z}\right)\left(\frac{1-\mathrm{e}^{-r T}}{T}+1\right)\right\}(1-p)
\end{aligned}
$$

[24].

Where the annual production of heat and cold is expressed by the respective formulae (Equations (20)-(22) [20]):

$$
\begin{gathered}
Q_{R c}=\frac{\left(N^{G T}+N^{T E}\right) \eta_{G} \tau_{R}}{\sigma_{R}} \\
Q_{R z}=\dot{Q}_{z} \tau_{z}=N_{z} \varepsilon_{z} \tau_{z}
\end{gathered}
$$

and the driving power of the compressor chiller is given the relation:

$$
N_{z}=x\left(N^{G T}+N^{T E}\right) \eta_{G} ; x \in\langle 0 ; 1\rangle
$$

where:

$a_{e l}, a_{c}, a_{z}, a_{f u e l}, a_{\mathrm{CO}}, a_{\mathrm{CO}}, a_{\mathrm{SO}_{2}}, a_{\mathrm{NO}_{X}}, a_{d u s t}, b_{\mathrm{CO}_{2}}$-terms of the exponents representing the changes in time of electricity, heat, cold, fuel prices, tariff charges on the environmental emissions, $\mathrm{CO}_{2}$ allowances; $e_{e l}^{t=0}, e_{f u e l}^{t=0} e_{c}^{t=0}, e_{z}^{t=0}, e_{\mathrm{CO}_{2}}^{t=0}, p_{\mathrm{CO}_{2}}^{t=0}$, etc. - initial prices of electricity, fuel, heat, cold, $\mathrm{CO}_{2}$ emission allowances, etc., $\dot{E}_{c h}$-stream of the chemical energy of fuel combustion in the gas turbine $\left(\dot{E}_{c h}=N^{G T} / \eta_{G T}^{\max }\right), J_{G-G}, J_{z}$-investment in the gas-gas system and chiller, $P_{\mathrm{CO}_{2}}, P_{\mathrm{CO}}, P_{\mathrm{NO}}, P_{\mathrm{SO}_{2}}$, $p_{\text {dust }}$ - specific charges on $\mathrm{CO}_{2}, \mathrm{CO}, \mathrm{NO}_{x}, \mathrm{SO}_{2}$, particulate matter emissions, $r$ - interest rate of capital 
investment, $x_{\text {sal, }, \text { ins }}$-factor applied to account for the cost of remuneration, taxes, insurance, etc. (usually the value $x_{\text {sal, }, \text {,ins }}$ is equal to around 0.25), $z$-discount coefficient (freezing coefficient) on investment $J$ at the instant when the investment is completed, $\delta_{\text {serv }}$-annual rate of fixed cost relative to the investment (cost of maintenance and overhaul of equipment), $\varepsilon_{z}=\dot{Q}_{z} / N_{z}$-thermal efficiency of chiller (in this analysis we adopted the use of a steam compressor chiller operating according to the Linde circulation; the ammonia forms the circulating medium in the system; in the calculations we adopted $\left.\varepsilon_{z}=3.2\right), \varepsilon_{e l}$-internal electric load of the system, $\eta_{G}$-efficiency of the electric generator, $\rho_{\mathrm{CO}_{2}}, \rho_{\mathrm{CO}}, \rho_{\mathrm{NO}_{x}}, \rho_{\mathrm{SO}_{2}}, \rho_{\text {dust }}-\mathrm{CO}_{2}, \mathrm{CO}, \mathrm{NO}_{\mathrm{x}}, \mathrm{SO}_{2}$, particulate matter emissions per specific value of the chemical energy of fuel, $\sigma_{R}=E_{e l, R} / Q_{R c}$-ratio of annual electricity production to annual heat production, $\tau_{R}$-annual duration of the operation of the trigeneration system (in the calculations, we assumed a value of $8424 \mathrm{~h}$; this accounts for the two-week downtime period projected within an annual operating schedule), $\tau_{z}$-annual operating time of a chiller expressed in hours.

From the conditions $N P V=0$ and $a_{z}=0$, we can derive the formula to represent the specific cost of cold production over the period of $T$ years:

$$
\begin{aligned}
& k_{z a v}=\frac{r \tau_{R}}{x\left(1+\frac{N^{T E}}{N G T}\right) \eta_{G} \varepsilon_{z} \tau_{z}\left(1-\mathrm{e}^{-r T}\right) \eta_{G T}^{\max }}\left\{\frac{e_{\text {fuel }}^{t=0}}{a_{f u e l}-r}\left[\mathrm{e}^{\left(a_{f u e l}-r\right) T}-1\right]+\right. \\
& +\frac{\rho_{\mathrm{CO}_{2}} p_{\mathrm{CO}_{2}}^{t=0}}{a_{\mathrm{CO}_{2}}-r}\left[\mathrm{e}^{\left(a_{\mathrm{CO}}-r\right) T}-1\right]+\frac{\rho_{\mathrm{CO}} \mathrm{CO}_{\mathrm{CO}}^{t=0}}{a_{\mathrm{CO}}-r}\left[\mathrm{e}^{\left(a_{\mathrm{CO}}-r\right) T}-1\right]+ \\
& +\frac{\rho_{\mathrm{NO}_{\mathrm{X}}} p_{\mathrm{NO}}^{t=0}}{a_{\mathrm{NO}}-r}\left[\mathrm{e}^{\left(a_{\mathrm{NO}_{\mathrm{X}}}-r\right) T}-1\right]+\frac{\rho_{\mathrm{SO}_{2}} p_{\mathrm{SO}_{2}}^{t=0}}{a_{\mathrm{SO}_{2}}-r}\left[\mathrm{e}^{\left(a_{\mathrm{SO}_{2}}-r\right) T}-1\right]+ \\
& \left.+\frac{\rho_{\text {dust }} p_{\text {dust }}^{t=0}}{a_{\text {dust }}-r}\left[\mathrm{e}^{\left(a_{\text {dust }}-r\right) T}-1\right]+\frac{\rho_{\mathrm{CO}_{2}} e_{\mathrm{CO}}^{t=0}}{b_{\mathrm{CO}_{2}}-r}\left[\mathrm{e}^{\left(b_{\mathrm{CO}_{2}}-r\right) T}-1\right]\right\}+ \\
& +\left(1+x_{\text {sal }, t, i n s}\right) \frac{\left(i_{G-G}+x i_{z}\right)}{x \varepsilon_{z} \tau_{z}} \delta_{\text {serv }}+\frac{r z\left(i_{G-G}+x i_{z}\right)}{x \varepsilon_{z} \tau_{z}\left(1-\mathrm{e}^{-r T}\right)}\left(\frac{1-\mathrm{e}^{-r T}}{T}+1\right)+ \\
& -\frac{r\left(\tau_{R}-x \tau_{z}\right)\left(1-\varepsilon_{e l}\right)}{x \varepsilon_{z} \tau_{z}\left(1-\mathrm{e}^{-r T}\right)} \frac{e_{e l}^{t=0}}{a_{e l}-r}\left[\mathrm{e}^{\left(a_{e l}-r\right) T}-1\right]-\frac{r \tau_{R}}{x \varepsilon_{z} \tau_{z} \sigma_{R}\left(1-\mathrm{e}^{-r T}\right)} \frac{e_{c}^{t=0}}{a_{c}-r}\left[\mathrm{e}^{\left(a_{c}-r\right) T}-1\right]
\end{aligned}
$$

[24],

where:

$i_{G-G}$-specific investment in the gas-gas engine (calculated per unit of electric capacity, $i_{G-G}=$ $J_{G-G} /\left[\left(N^{G T}+N^{T E}\right) \eta_{G}\right], i_{z}$-specific investment in the chiller (calculated per unit of electric capacity), $i_{z}=J_{z} / N_{z}$ (the calculations account for two alternatives of this investment; $i_{z}=0.5 i_{G-G}, i_{z}=0.7 i_{G-G}$ ).

The revenues from the sales of electricity and heat (Formulae (19) and (23)) form the avoided cost of cold production.

In addition to capital expenditure, the energy efficiency of the gas turbine, chiller power and its operating times, prices of electricity, fuel and heat, one of the factors that has a significant impact on the unit cost of production of cold $k_{z a v}$ is associated with the price of carbon dioxide emission allowances $e_{\mathrm{CO}_{2}}$. It fluctuates to a considerable degree, even on a week to week basis, as it is often a speculative price. In the calculations, for its comparative purposes, its dual alternative value was adopted. In one of the alternatives, it was assumed to be equal to the so-called reference settlement price $e_{\mathrm{CO}_{2}}^{r e f}=$ $20.38 € / \mathrm{MgCO}_{\mathrm{C} 2}\left(\sim 90 \mathrm{PLN} / \mathrm{MgCO}_{\mathrm{C} 2}\right.$ ), which are derived on the basis of the Directive $2003 / 87 / \mathrm{EC}$ and later 2009/29/EC imposes the EU ETS (European Union Emission Trading Scheme) emissions trading system, and in the other $e_{\mathrm{CO}_{2}}=20 \mathrm{PLN} / \mathrm{MgCO}_{\mathrm{C}}$.

The formula in (23) also determines the specific costs of the production of cold in all three alternatives of the systems operating with heat regeneration, i.e., for systems with an applied regenerative heat exchanger: (1) only in a gas turbine, (2) only in a turboexpander and (3) in a system in which regenerative heat exchangers are employed simultaneously in the gas turbine and in the turboexpander. As a result, in Formula (23), higher values of the specific values of investment are obtained for the various power ratios $N^{T E} / N^{G T}$ in the particular alternatives of the system design derived from thermodynamic analysis. 
Figures 7-12 present the results of the calculations of the specific cost of cold production in a system with a compressor chiller involving a variety of alternative designs. Table 1 contains a summary of the input data adopted for calculations for individual curves presented in Figures 7-12.

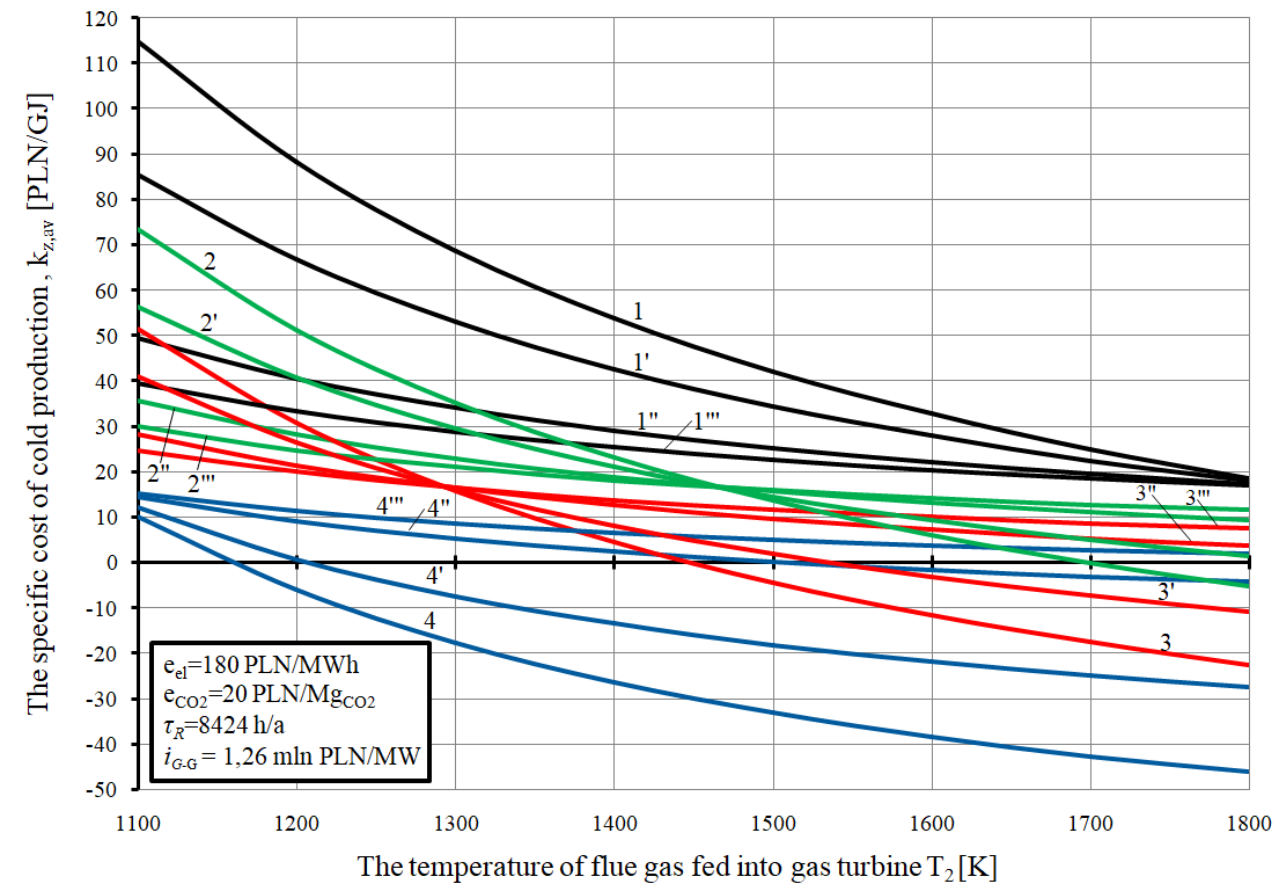

Figure 7. Specific values of production of cold $k_{z, a v}$ in a gas-gas system comprising a turboexpander excluding heat regeneration in the compressor chiller in the function of the temperature $T_{2}$ of flue gas fed into gas turbine.

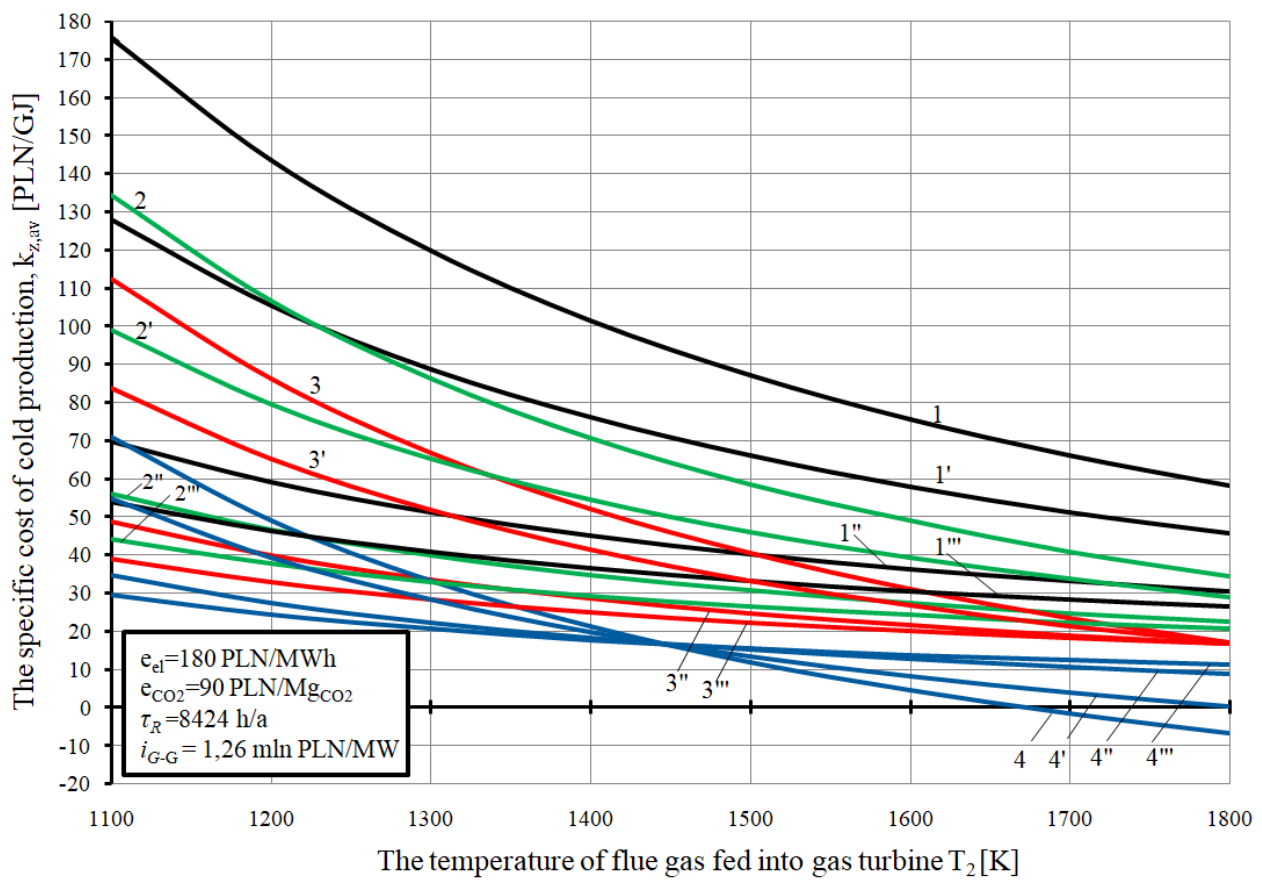

Figure 8. Specific values of production of cold $k_{z, a v}$ in a gas-gas system comprising a turboexpander excluding heat regeneration in the function of the temperature $T_{2}$ of flue gas fed into gas turbine. 


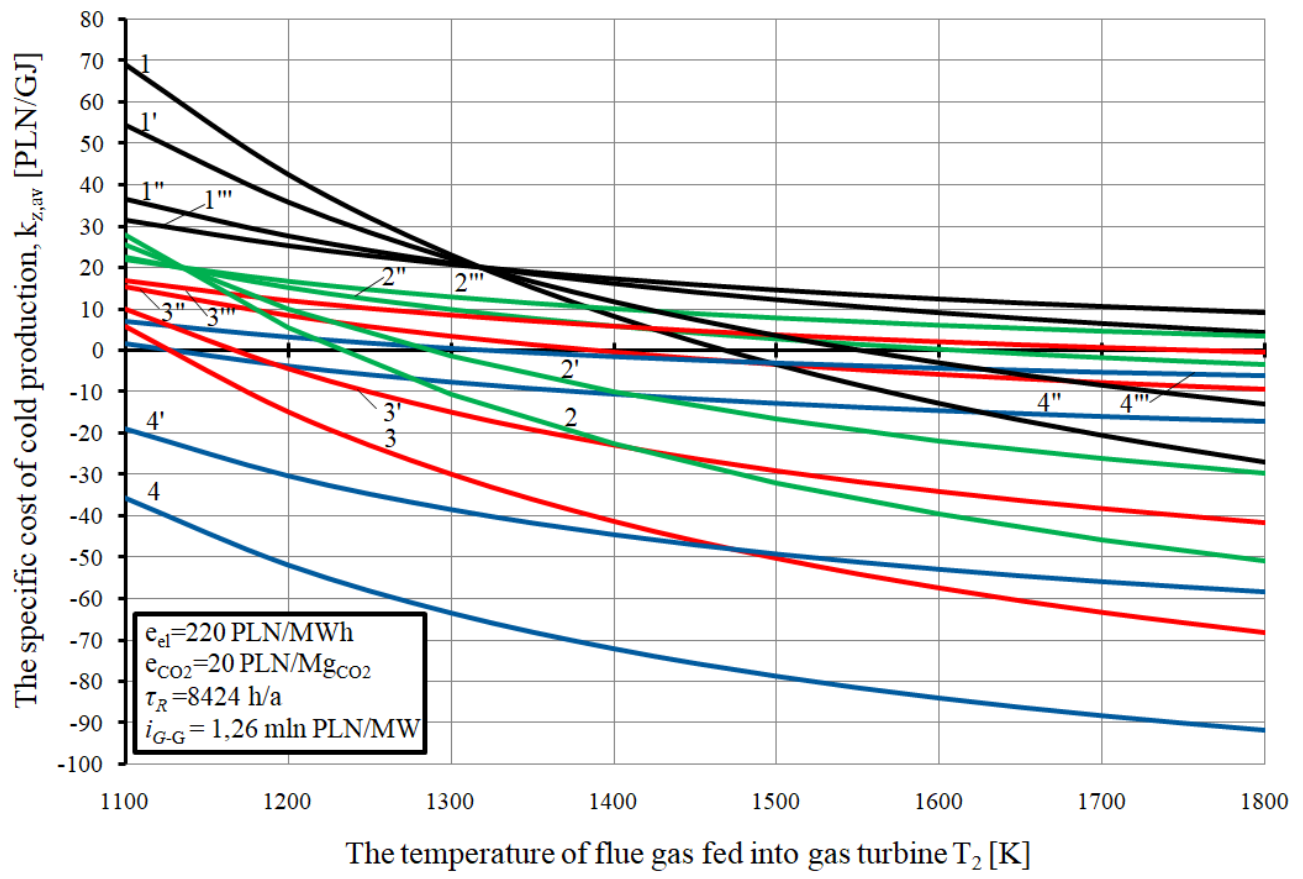

Figure 9. Specific values of production of cold $k_{z, a v}$ in a gas-gas system comprising a turboexpander and a compressor chiller excluding heat regeneration in the function of the temperature of flue gas $T_{2}$ fed into gas turbine.

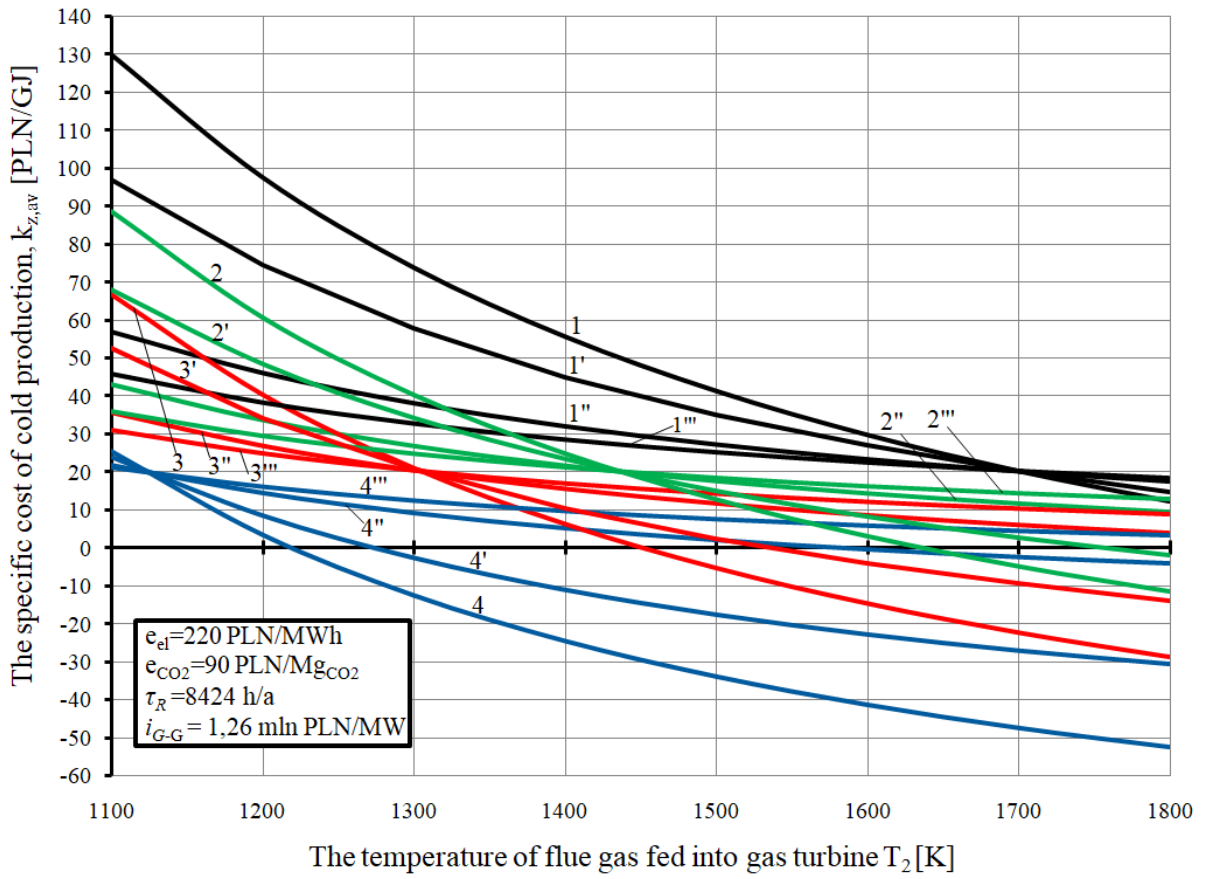

Figure 10. Values of specific values of production of cold $k_{z, a v}$ in a gas-gas system comprising a turboexpander and compressor chiller excluding heat regeneration in the function of the temperature of flue gas $T_{2}$ fed into gas turbine. 


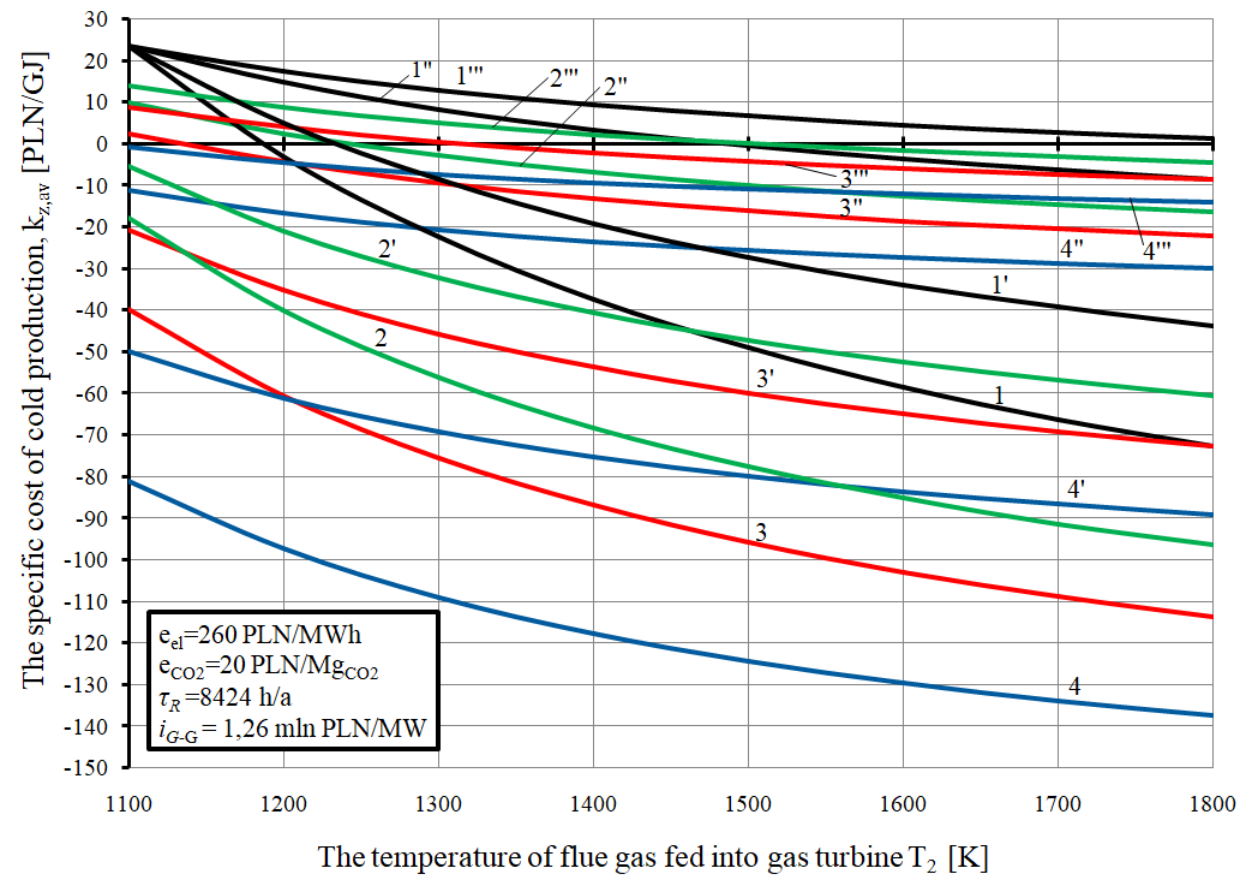

Figure 11. Values of specific values of production of cold $k_{z, a v}$ in a gas-gas system comprising a turboexpander and compressor chiller excluding heat regeneration in the function of the temperature of flue gas $T_{2}$ fed into gas turbine.

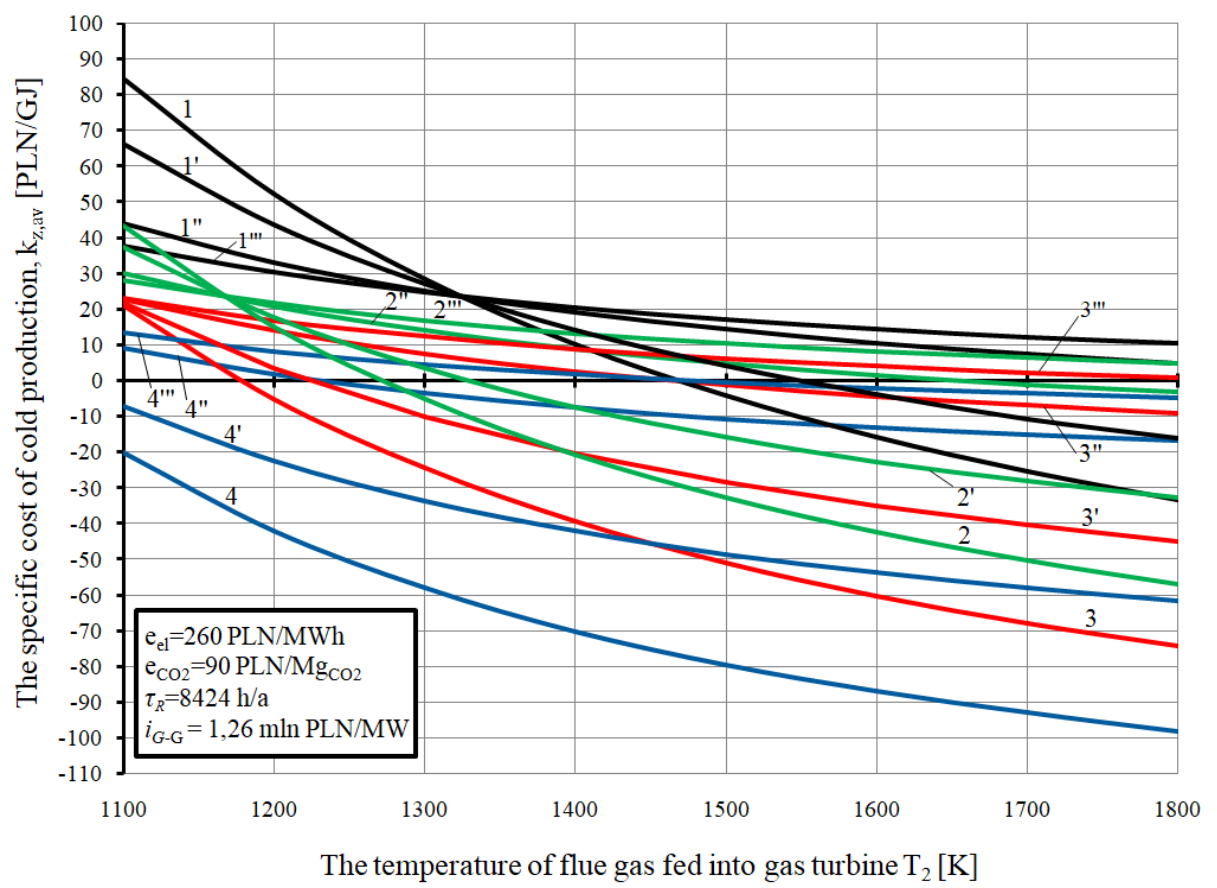

Figure 12. Values of specific values of production of cold $k_{z, a v}$ in a gas-gas system comprising a turboexpander and compressor chiller excluding heat regeneration in the function of the temperature of flue gas $T_{2}$ fed into gas turbine. 
Table 1. Input data for analysis.

\begin{tabular}{ccccccccc}
\hline Symbols & $\mathbf{1}$ & $\mathbf{1}^{\prime}$ & $\mathbf{1}^{\prime \prime}$ & $\mathbf{1}^{\prime \prime \prime}$ & $\mathbf{2}$ & $\mathbf{2}^{\prime}$ & $\mathbf{2}^{\prime \prime}$ & $\mathbf{2}^{\prime \prime \prime}$ \\
\hline $\mathrm{e}_{\mathrm{c}}, \mathrm{PLN} / \mathrm{GJ}$ & 50 & 50 & 50 & 50 & 70 & 70 & 70 & 70 \\
$\mathrm{e}_{\text {fuel }}, \mathrm{PLN} / \mathrm{GJ}$ & 24 & 24 & 24 & 24 & 24 & 24 & 24 & 24 \\
$i_{z}, \mathrm{PLN} / \mathrm{MW}$ & $0.5 i_{G-G}$ & $0.7 i_{G-G}$ & $0.5 i_{G-G}$ & $0.7 i_{G-G}$ & $0.5 i_{G-G}$ & $0.7 i_{G-G}$ & $0.5 i_{G-G}$ & $0.7 i_{G-G}$ \\
$\tau_{z}, \mathrm{~h} / \mathrm{a}$ & $0.7 \tau_{R}$ & $\tau_{z}=\tau_{R}$ & $0.7 \tau_{R}$ & $\tau_{z}=\tau_{R}$ & $0.7 \tau_{R}$ & $\tau_{z}=\tau_{R}$ & $0.7 \tau_{R}$ & $\tau_{z}=\tau_{R}$ \\
$\mathrm{x}, \%$ & 0,1 & 0,1 & 0,3 & 0,3 & 0,1 & 0,1 & 0,3 & 0,3 \\
\hline & $\mathbf{3}$ & $\mathbf{3}^{\prime}$ & $\mathbf{3}^{\prime \prime}$ & $\mathbf{3}^{\prime \prime \prime}$ & $\mathbf{4}$ & $\mathbf{4}^{\prime}$ & $\mathbf{4}^{\prime \prime}$ & $\mathbf{4}^{\prime \prime \prime}$ \\
\hline $\mathrm{e}_{\mathrm{c}}, \mathrm{PLN} / \mathrm{GJ}$ & 50 & 50 & 50 & 50 & 70 & 70 & 70 & 70 \\
$\mathrm{e}_{\text {fuel }}, \mathrm{PLN} / \mathrm{GJ}$ & 20 & 20 & 20 & 20 & 20 & 20 & 20 & 20 \\
$i_{z}, \mathrm{PLN} / \mathrm{MW}$ & $0.5 i_{G-G}$ & $0.7 i_{G-G}$ & $0.5 i_{G-G}$ & $0.7 i_{G-G}$ & $0.5 i_{G-G}$ & $0.7 i_{G-G}$ & $0.5 i_{G-G}$ & $0.7 i_{G-G}$ \\
$\tau_{z}, \mathrm{~h} / \mathrm{a}$ & $0.7 \tau_{R}$ & $\tau_{z}=\tau_{R}$ & $0.7 \tau_{R}$ & $\tau_{z}=\tau_{R}$ & $0.7 \tau_{R}$ & $\tau_{z}=\tau_{R}$ & $0.7 \tau_{R}$ & $\tau_{z}=\tau_{R}$ \\
x, \% & 0,1 & 0,1 & 0,3 & 0,3 & 0,1 & 0,1 & 0,3 & 0,3 \\
\hline
\end{tabular}

where:

$\mathrm{e}_{\mathrm{c}}$-prices of heat in PLN (Polish Zloty) per GJ, $\mathrm{e}_{\text {fuel }}$-prices of fuel in PLN (Polish Zloty) per GJ, $i_{z}$-specific investment in the chiller (calculated per unit of electric capacity) in PLN (Polish Zloty) per $\mathrm{MW}, \tau_{z}$-annual operating time of a chiller expressed in hours per year.

As we can see from Figures 7-12, the specific cost of cold production $k_{z, a v}$ gets higher as a consequence of the decrease in the price of electricity and the higher prices of fuel and $\mathrm{CO}_{2}$ emission allowances, and along with the greater cost of investment. As a result of increasing the value of $x$, and thus increasing the production of cold in the system (Formulae (21), (22)), this cost increases as well. As a consequence, the electricity production decreases, and thus the revenues from its sale decreases as well, which forms the avoided cost associated with the production of cold.

On the basis of the analysis of the performance of the system, it is also feasible to establish an answer to the question: to what extent does the use of heat regeneration in the turboexpander in Figure 4, leading to an increase in the electricity production, affect the value of specific cost of production of cold? Under the assumption of the fact that the investment associated with a system comprising regeneration is the same as the cost for a system excluding a regenerative heat exchanger, it appears that the decrease of this cost is negligible, as it does not exceed 0.5 PLN/GJ in the whole range of variations in temperature $T_{2}$. Therefore, the curves representing the specific cost presented in Figures 7-12 for a system without regeneration overlap with the curves representing a system with regenerative heat exchanger. Therefore, we can conclude that the design of a gas-gas system including a turboexpander including a regenerative heat exchanger leads to a zero value of the increase in the specific cost of cold production in the best case, for the case when we take into account the increase in expenditures in the economic calculation associated with the capital costs (depreciation and financial cost) as well as cost of the normal operation (repairs and maintenance). Therefore, the construction of a system with heat regeneration is completely economically unfeasible.

The analysis of the specific cost of cold production presented in Figures 7-12 also demonstrates, as already noted above, that it is more profitable to produce only electricity and heat in the system [1] than combine this production with additional production of cold. Therefore, it is important to answer the question: what should be the price of cold so that it would be profitable to produce it.

Condition of Economic Profitability of Application of Compressor Chiller in the Trigeneration System comprising Gas-Gas Engine

A necessary condition for the economic feasibility of using a compressor chiller in the trigeneration system is related to the fact that the value of NPV derived from its operation (Formula (19)) is greater than the profit gained from the operation of the system in which only electricity and heat is produced 
(in Formulae (19) and (22) zero need to then substitute $N_{z}$ and $J_{z}$ ). This condition is therefore expressed by the relation:

$$
\begin{aligned}
& Q_{R z} \frac{e_{z \min }^{t=0}}{a_{z}-r}\left[\mathrm{e}^{\left(a_{z}-r\right) T}-1\right] \geq N_{z} \tau_{z}\left(1-\varepsilon_{e l}\right) \frac{e_{e l}^{t=0}}{a_{e l}-r}\left[\mathrm{e}^{\left(a_{e l}-r\right) T}-1\right]+ \\
& +\left(1+x_{\text {sal,t,ins }}\right) J_{z}\left(1-\mathrm{e}^{-r T}\right) \frac{\delta_{\text {serv }}}{r}+z J_{z}\left(\frac{1-\mathrm{e}^{-r T}}{T}+1\right)
\end{aligned}
$$

By application of Equation (15), we can derive the final form of the condition from (24) to represent the mean price $\left(a_{z}=0\right)$ of the sales of cold over a period of $T$ years:

$$
e_{z a v}^{\min } \geq \frac{\left(1-\varepsilon_{e l}\right)}{\varepsilon_{z}} e_{\text {elav }}+\left(1+x_{\text {sal, }, \text {,ins }}\right) \frac{i_{z} \delta_{\text {serv }}}{\varepsilon_{z} \tau_{z}}+\frac{r z i_{z}}{\varepsilon_{z} \tau_{z}\left(1-\mathrm{e}^{-r T}\right)}\left(\frac{1-\mathrm{e}^{-r T}}{T}+1\right)
$$

where the mean price $e_{\text {elav }}$ of the sales of electricity over the period of $T$ years is expressed by the formula:

$$
e_{\text {elav }}=\frac{1}{T} \int_{0}^{T} e_{e l}^{t=0} \mathrm{e}^{a_{e l} t} d t=\frac{e_{e l}^{t=0}}{T a_{e l}}\left(\mathrm{e}^{a_{e l} T}-1\right)
$$

However, it should be very strongly emphasized that the condition of the economic feasibility of the application of the trigeneration system is not related to fulfilling of the relationship (25), but to the fact that the following relation is fulfilled:

$$
e_{z a v} \geq k_{z a v}
$$

which means that the sales price of the production of cold should be at least not lower from the specific cost associated with its production. This is due to the possible condition in which the price $e_{z a v}^{\min }$ (Formula (25)) is lower than the cost $k_{z a v}$ (Formula(26)). This can occur when the electricity price assumes a relatively low price, generally associated with the large cost $k_{z}$ av -Figure 13. The price $e_{z a v}^{\min }$ is, in contrast to the cost $k_{z} a v$, derived on the basis of excluding the cost of fuel and environmental charges, which are removed in the process of subtracting profit expressed by $N P V$, i.e., when the profit $N P V$ derived from the operation of the trigeneration system we subtract the value of $N P V$ gained from the cogeneration system (Formula (25)).

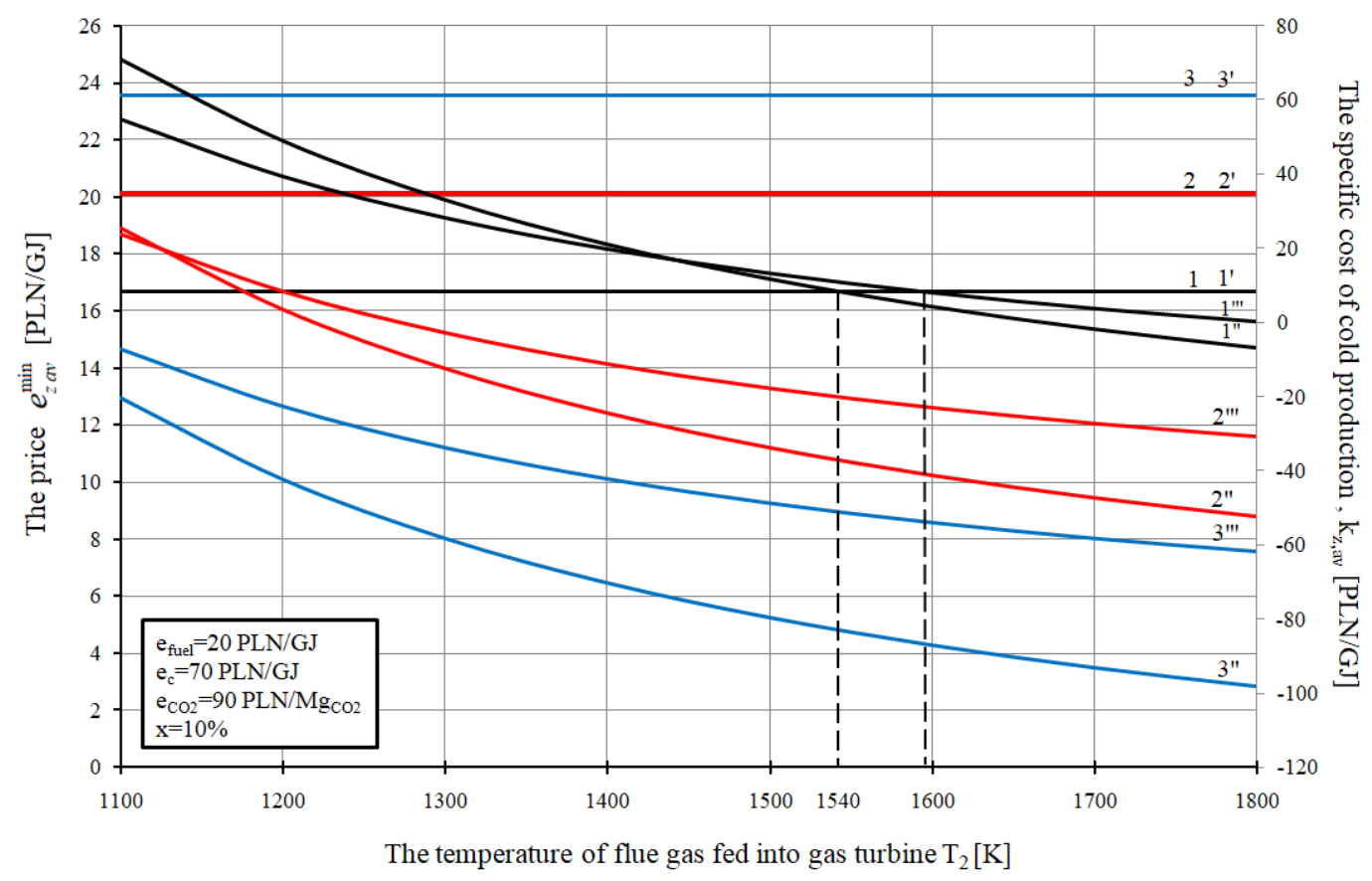

Figure 13. Values of $e_{z a v}^{\min }, k_{z a v}$ in the function of the temperatures of the gas fed into the gas turbine $T_{2}$. 
The courses of the exemplary curves $e_{z a v}^{\min }$ and $k_{z}$ av are presented in Figure 13. Table 2 contains a summary of the data taken for its calculations.

Table 2. Summary of input data.

\begin{tabular}{ccccccc}
\hline $\mathbf{e}_{\text {el }}$, & \multirow{2}{*}{$\mathbf{1 8 0}$} & $\mathbf{1 8 0}$ & $\mathbf{2 2 0}$ & $\mathbf{2 2 0}$ & $\mathbf{2 6 0}$ & $\mathbf{2 6 0}$ \\
$\mathbf{P L N} / \mathbf{M W h}$ & & & & & & \\
\hline $\mathrm{i}_{\mathrm{z}}, \mathrm{PLN} / \mathrm{MW}$ & $0.5 i_{G-G}$ & $0.7 i_{G-G}$ & $0.5 i_{G-G}$ & $0.7 i_{G-G}$ & $0.5 i_{G-G}$ & $0.7 i_{G-G}$ \\
$\tau_{\mathrm{z}}, \mathrm{h} / \mathrm{a}$ & $0.7 \tau_{R}$ & $\tau_{z}=\tau_{R}$ & $0.7 \tau_{R}$ & $\tau_{z}=\tau_{R}$ & $0.7 \tau_{R}$ & $\tau_{z}=\tau_{R}$ \\
$e_{z a v}^{\mathrm{min}}, \mathrm{PLN} / \mathrm{GJ}$ & 1 & $1^{\prime}$ & 2 & $2^{\prime}$ & 3 & $3^{\prime}$ \\
$k_{z, a v}, \mathrm{PLN} / \mathrm{GJ}$ & $1^{\prime \prime}$ & $1^{\prime \prime \prime}$ & $2^{\prime \prime}$ & $2^{\prime \prime \prime}$ & $3^{\prime \prime}$ & $3^{\prime \prime \prime}$ \\
\hline
\end{tabular}

\subsection{Analysis of System Comprising an Absorption Chiller}

The basic advantage of the absorption chiller is associated with the fact that such a system does not include a compressor, and therefore electric power is not applied for the purposes of driving it, while its operating principle is based on the use of the waste enthalpy of the flue gas from a gas-gas engine, Figure 1c. As a result of this, in comparison to the system comprising a compressor chiller, a greater volume of electricity is produced, i.e., the noblest, and thus the most valuable, and the most expensive form of energy, and the revenue from its sale is related to the cost of avoided cold production. The disadvantage of the system comprising an absorption chiller is its low refrigerating power. The temperature of the flue gas which constitutes the source of heat in the desorber of the absorption chiller needs to be higher than the temperature of the ammonia solution that is fed into it. This temperature is equal to around $T_{8},=395 \mathrm{~K}$, whereas the temperature $T_{8}$ of the flue gas fed into the desorber is in the range from around 458 to $524 \mathrm{~K}$ (this temperature increases along with the temperature of the combustion of gas $T_{2}$ in the combustion chamber $\mathrm{K}$ of the gas turbine; value $T_{8}=$ $458 \mathrm{~K}$ corresponds to the temperature $T_{2}=1100 \mathrm{~K}$, whereas the temperature $T_{8}=524 \mathrm{~K}$ corresponds to $T_{2}=1800 \mathrm{~K}$ [1]). The disposable span of the temperatures, i.e., the range that is feasible for application to drive the chiller exhaust, is therefore relatively small and is found from about 63 to about $130 \mathrm{~K}$, which involves a relatively small value of the heat flux $\dot{Q}_{z}$ extracted from the cooling elements in the system in comparison to the case when the compressor chiller is applied. The refrigerating power $\dot{Q}_{z}$ of the absorption chiller is therefore expressed by the equation:

$$
\dot{Q}_{z}=\left(\dot{C}_{f g}+\dot{C}_{a i r}\right)\left(T_{8}-395\right) \varepsilon_{z a b s}
$$

and the annual production of cold is expressed by the relation:

$$
Q_{R z}=\dot{Q}_{z} \tau_{z}=\left(\dot{C}_{f g}+\dot{C}_{a i r}\right)\left(T_{8}-395\right) \varepsilon_{z a b s} \tau_{z}
$$

where the energy balances of the circulations of the gas turbine and turboexpander can be applied to the fluxes of thermal capacities $\dot{C}_{f g}, \dot{C}_{a i r}$ of the circulating medium flow in the Joule circulation through the gas turbine $G T$ and turboexpander TE:

$$
\begin{aligned}
& N^{G T}=\dot{C}_{f g}\left[\eta_{m}\left(T_{2}-T_{3}\right)-\frac{\left(T_{1}-T_{a m b}\right)}{\eta_{m}}\right] \\
& N^{T E}=\dot{C}_{a i r}\left[\eta_{m}\left(T_{6}-T_{7}\right)-\frac{\left(T_{5}-T_{a m b}\right)}{\eta_{m}}\right]
\end{aligned}
$$

where:

$N^{G T}, N^{T E}$ - powers of the gas turbine $G T$ and turboexpander $T E$, Figure 3, $T_{1}$-temperature of circulating medium behind the compressor $C_{G T}$, Figure 3, $T_{2}$-temperature of circulating medium fed at the inlet to GT, Figure 3, $T_{3}$-temperature of circulating medium extracted from GT, Figure 3, 
$T_{5}$-temperature of circulating medium behind the compressor $C_{T E^{\prime}}$, Figure $3, T_{6}$-temperature of circulating medium fed at the inlet to $T E$, Figure $3, T_{7}$-temperature of circulating medium extracted from turboexpander $T E$, Figure $3, T_{8}$-temperature of heating medium fed at the inlet to the chiller, Figure $3, \varepsilon_{z}$ abs -thermal efficiency of cooling process of the absorption chiller; it was assumed to be equal to $\varepsilon_{z}$ abs $=0.37, \tau_{z}$-annual operating time of the chiller expressed in hours.

Figure 14 contains the results of thermodynamic calculations concerned with the value of the ratio of annual heat production to annual production of electricity $Q_{R c} / E_{e l, R}$ and the value of the ratio of annual cold production to annual production of electricity $Q_{R z} / E_{e l, R}$ in the system with a thermal chiller. We can note here that the ratio $Q_{R c} / E_{e l, R}$ in the system with the compressor chiller is about three times higher, as the enthalpy of flue gas extracted from the engine is applied only for heat production, Figures 1 and 2. This ratio, just as for the system with an thermal chiller, it also depends on the temperature $T_{2}$ and decreases from $1 / 2.16$ to $1 / 3.77$ in the range $T_{2} \in\langle 1100 ; 1800 \mathrm{~K}\rangle$.In addition, the cooling power of the system comprising the compressor chiller, which is relative to the value of $x$ (Formula (22)), is significantly higher as a result of the driving of the chiller by electricity (formula (21)). In addition, its thermal efficiency is equal to $\varepsilon_{z}=3.2$ whereas for the absorption chiller, this is $\varepsilon_{z a b s}=0.37$. For $\mathrm{x} \approx 0.1$, the cooling power of the compressor chiller is already equal to the capacity of the absorption chiller. As a consequence, the revenues gained from the sales of heat in the system with the compressor chiller-Figures 1 and 2-is about three times larger than the revenues resulting from the application of the system comprising an absorption chiller. However, in a system comprising a compressor chiller, the revenues from the sales of electricity are decreased to the same extent that the value of $x$ increases to meet the demand of the power to drive the chiller.

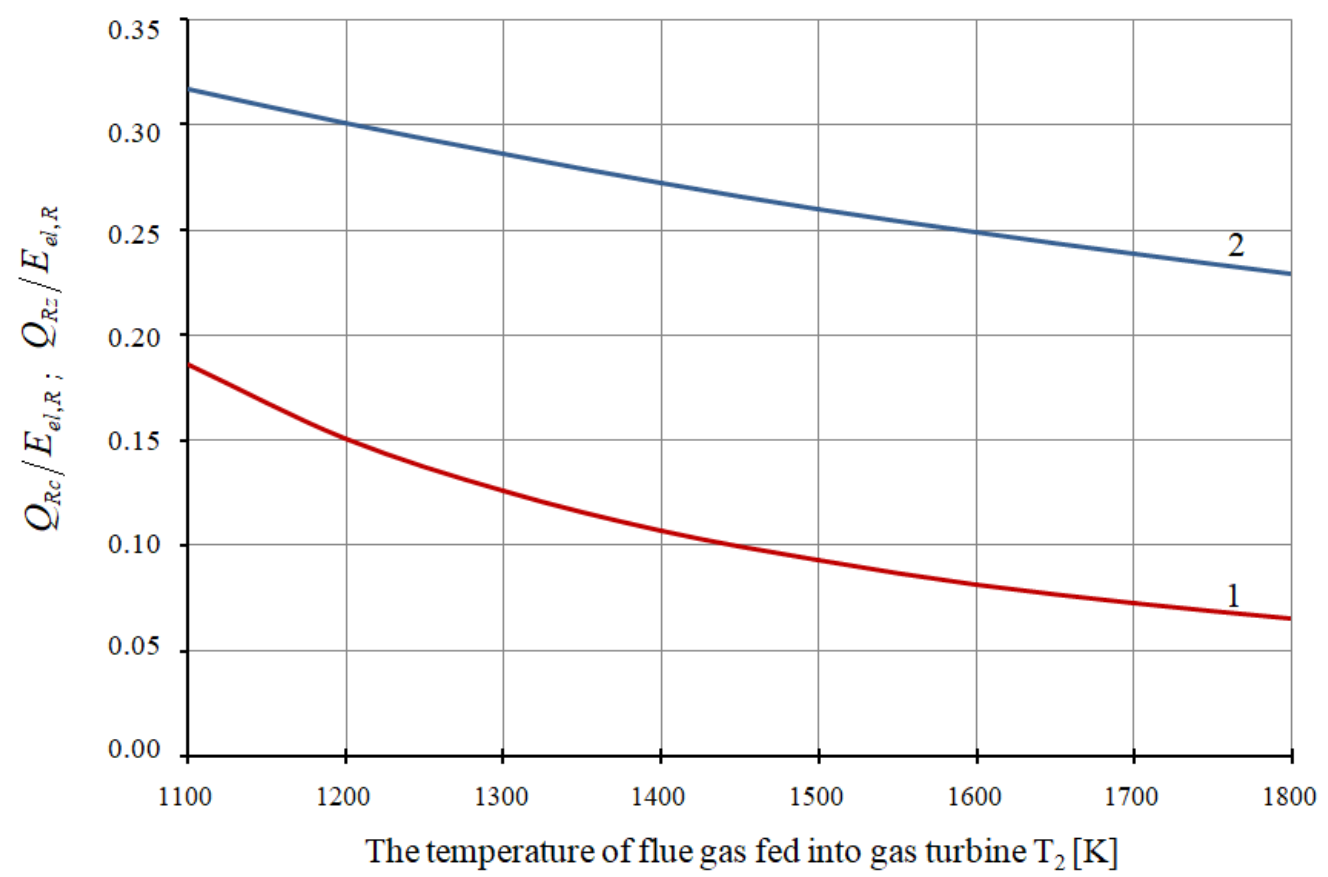

Figure 14. Ratios of annual production of heat and cold to the annual production of electricity in the gas-gas system comprising a absorption chiller in the function of temperature of flue gas fed into gas turbine $T_{2}$.

$$
\left(1: Q_{R c} / E_{e l, R} ; 2: Q_{R z} / E_{e l, R}\right),
$$


The total discounted $N P V$ gained from the operation of the trigeneration system comprising an thermal absorption chiller is expressed by the relation $[23,24]$ :

$$
\begin{aligned}
& N P V=\left\{\left[\left(N^{G T}+N^{T E}\right) \eta_{G} \tau_{R}\right]\left(1-\varepsilon_{e l}\right) \frac{e_{e l}^{t=0}}{a_{e l}-r}\left[\mathrm{e}^{\left(a_{e l}-r\right) T}-1\right]+Q_{R c} \frac{e_{c}^{t=0}}{a_{c}-r}\left[\mathrm{e}^{\left(a_{c}-r\right) T}-1\right]+\right. \\
& +Q_{R z} \frac{e_{z}^{t=0}}{a_{z}-r}\left[\mathrm{e}^{\left(a_{z}-r\right) T}-1\right]-\dot{E}_{c h} \tau_{R}\left\{\frac{e_{f u e l}^{t=0}}{a_{f u l}-r}\left[\mathrm{e}^{\left(a_{f u e l}-r\right) T}-1\right]+\right. \\
& +\frac{\rho_{\mathrm{CO}_{2}} p_{\mathrm{CO}_{2}}^{t=0}}{a_{\mathrm{CO}_{2}}-r}\left[\mathrm{e}^{\left(a_{\mathrm{CO}_{2}}-r\right) T}-1\right]+\frac{\rho_{\mathrm{CO}} t_{\mathrm{CO}}^{t=0}}{a_{\mathrm{CO}}-r}\left[\mathrm{e}^{\left(a_{\mathrm{CO}}-r\right) T}-1\right]+ \\
& +\frac{\rho_{\mathrm{NO}_{\mathrm{X}}} p_{\mathrm{NO}}^{t=0}}{a_{\mathrm{NO}_{\mathrm{X}}}-r}\left[\mathrm{e}^{\left(a_{\mathrm{NO}_{\mathrm{X}}}-r\right) T}-1\right]+\frac{\rho_{\mathrm{SO}_{2}} p_{\mathrm{SO}_{2}}^{t=0}}{a_{\mathrm{SO}_{2}-r}}\left[\mathrm{e}^{\left(a_{\mathrm{SO}_{2}}-r\right) T}-1\right]+ \\
& \left.+\frac{\rho_{\text {dust }} p_{\text {dust }}^{t=0}}{a_{\text {dust }}-r}\left[\mathrm{e}^{\left(a_{\text {dust }}-r\right) T}-1\right]+\frac{\rho_{\mathrm{CO}_{2}} e_{\mathrm{CO}}^{t=0}}{b_{\mathrm{CO}_{2}}-r}\left[\mathrm{e}^{\left(b_{\mathrm{CO}_{2}}-r\right) T}-1\right]\right\}+ \\
& \left.-\left(1+x_{\text {sal }, \text { t,ins }}\right)\left(J_{G-G}+J_{z}\right)\left(1-\mathrm{e}^{-r T}\right) \frac{\delta_{\text {serv }}}{r}-z\left(J_{G-G}+J_{z}\right)\left(\frac{1-\mathrm{e}^{-r T}}{T}+1\right)\right\}(1-p)
\end{aligned}
$$

whereas the specific cost of production of cold based on the conditions that $N P V=0$ and $a_{z}=0$ is given by the formula:

$$
\begin{aligned}
& k_{z a v}=\frac{r \tau_{R}}{A \eta_{T G}^{\max } \varepsilon_{z a b s} \tau_{z}\left(1-\mathrm{e}^{-r T}\right)}\left\{\frac{e_{f u e l}^{t=0}}{a_{f u e l}-r}\left[\mathrm{e}^{\left(a_{f u e l}-r\right) T}-1\right]+\frac{\rho_{\mathrm{CO}_{2}} p_{\mathrm{CO}_{2}}^{t=0}}{a_{\mathrm{CO}_{2}}-r}\left[\mathrm{e}^{\left(a_{\mathrm{CO}_{2}}-r\right) T}-1\right]+\right. \\
& +\frac{\rho_{\mathrm{CO}} p_{\mathrm{CO}}^{t=0}}{a_{\mathrm{CO}}-r}\left[\mathrm{e}^{\left(a_{\mathrm{CO}}-r\right) T}-1\right]+\frac{\rho_{\mathrm{NO}_{X}} p_{N O_{\mathrm{X}}}^{t=0}}{a_{\mathrm{NO}_{X}}^{-r}}\left[\mathrm{e}^{\left(a_{\mathrm{NO}_{X}}-r\right) T}-1\right]+\frac{\rho_{\mathrm{SO}_{2}} p_{\mathrm{SO}_{2}}^{t=0}}{a_{\mathrm{SO}_{2}}-r}\left[\mathrm{e}^{\left(a_{\mathrm{SO}_{2}}-r\right) T}-1\right]+ \\
& \left.+\frac{\rho_{\text {dust }} p_{\text {dust }}^{t=0}}{a_{\text {dust }}-r}\left[\mathrm{e}^{\left(a_{\text {dust }}-r\right) T}-1\right]+\frac{\rho_{\mathrm{CO}_{2}} e_{\mathrm{CO}_{2}}^{t=0}}{b_{\mathrm{CO}_{2}}-r}\left[\mathrm{e}^{\left(b_{\mathrm{CO}_{2}}-r\right) T}-1\right]\right\}+
\end{aligned}
$$

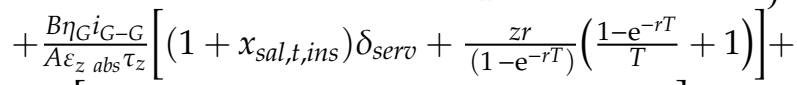

$$
\begin{aligned}
& +\frac{i_{z}}{\tau_{z}}\left[\left(1+x_{\text {sal }, t, i n s}\right) \delta_{\text {serv }}+\frac{z r}{\left(1-\mathrm{e}^{-r T}\right)}\left(\frac{1-\mathrm{e}^{-r T}}{T}+1\right)\right]+
\end{aligned}
$$

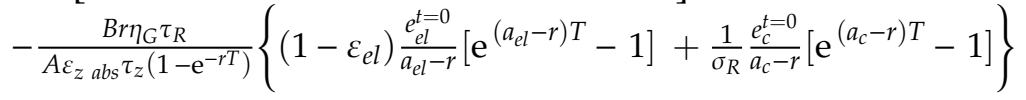

where:

$$
\begin{gathered}
A=\left[\frac{\eta_{m}}{\eta_{m}^{2}\left(T_{2}-T_{3}\right)-\left(T_{1}-T_{a m b}\right)}+\frac{N^{T E}}{N^{G T}} \frac{\eta_{m}}{\eta_{m}^{2}\left(T_{6}-T_{7}\right)-\left(T_{5}-T_{a m b}\right)}\right]\left(T_{8}-395\right) \\
B=\left(1+\frac{N^{T E}}{N^{G T}}\right) \\
i_{z}=\frac{J_{z}}{\dot{Q}_{z}}
\end{gathered}
$$

Figures 15-20 contain the results of thermodynamic calculations concerned with the specific cost of production of cold $k_{z, a v}$ for the gas-gas system comprising a turboexpander excluding a regenerative heat exchanger. Table 3 contains a summary of the input data adopted for calculations for individual curves presented in Figures 15-20. 


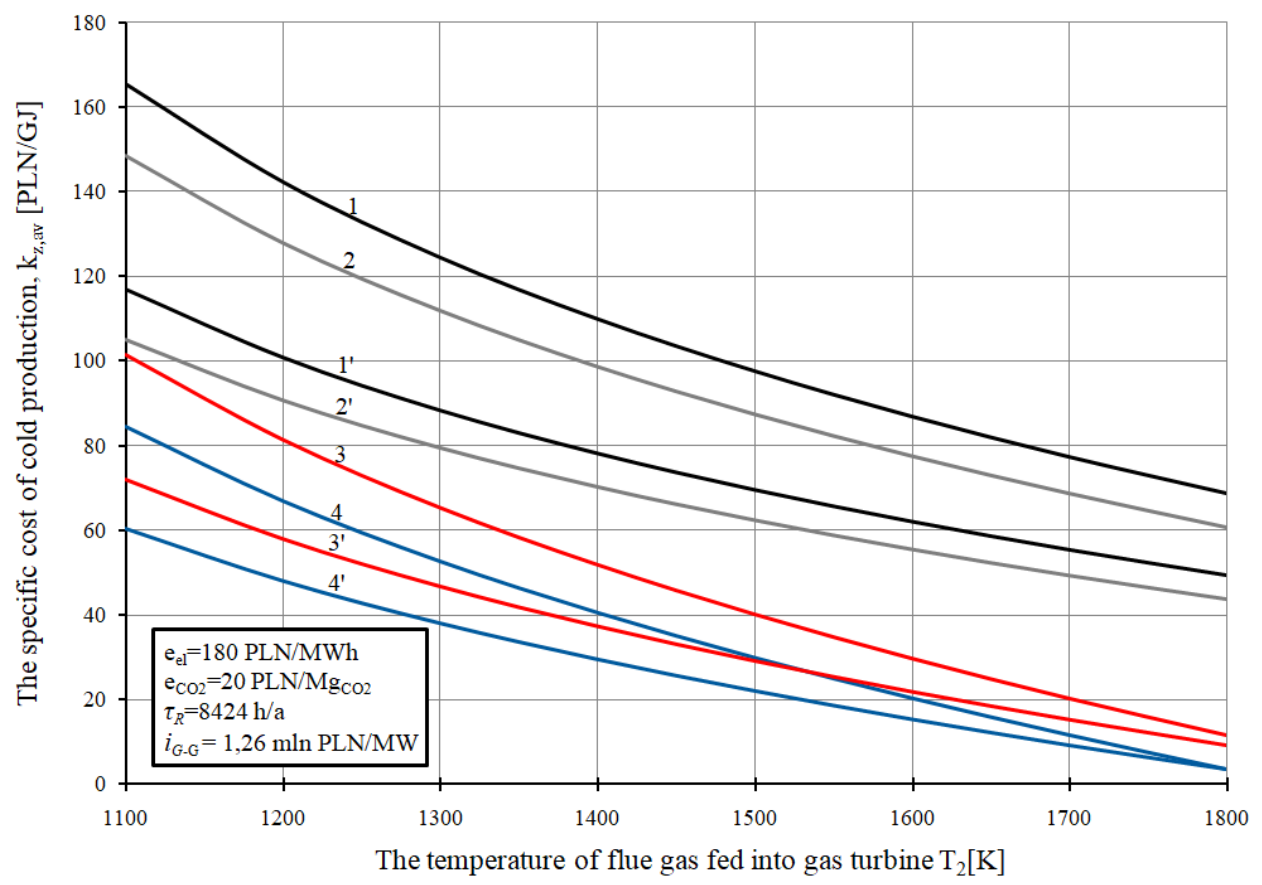

Figure 15. Specific value of cost of cold production $k_{z, a v}$ in the gas-gas system with turboexpander excluding regenerative heat exchanger with absorption chiller in the function of the temperature of gas fed into the gas turbine $T_{2}$.

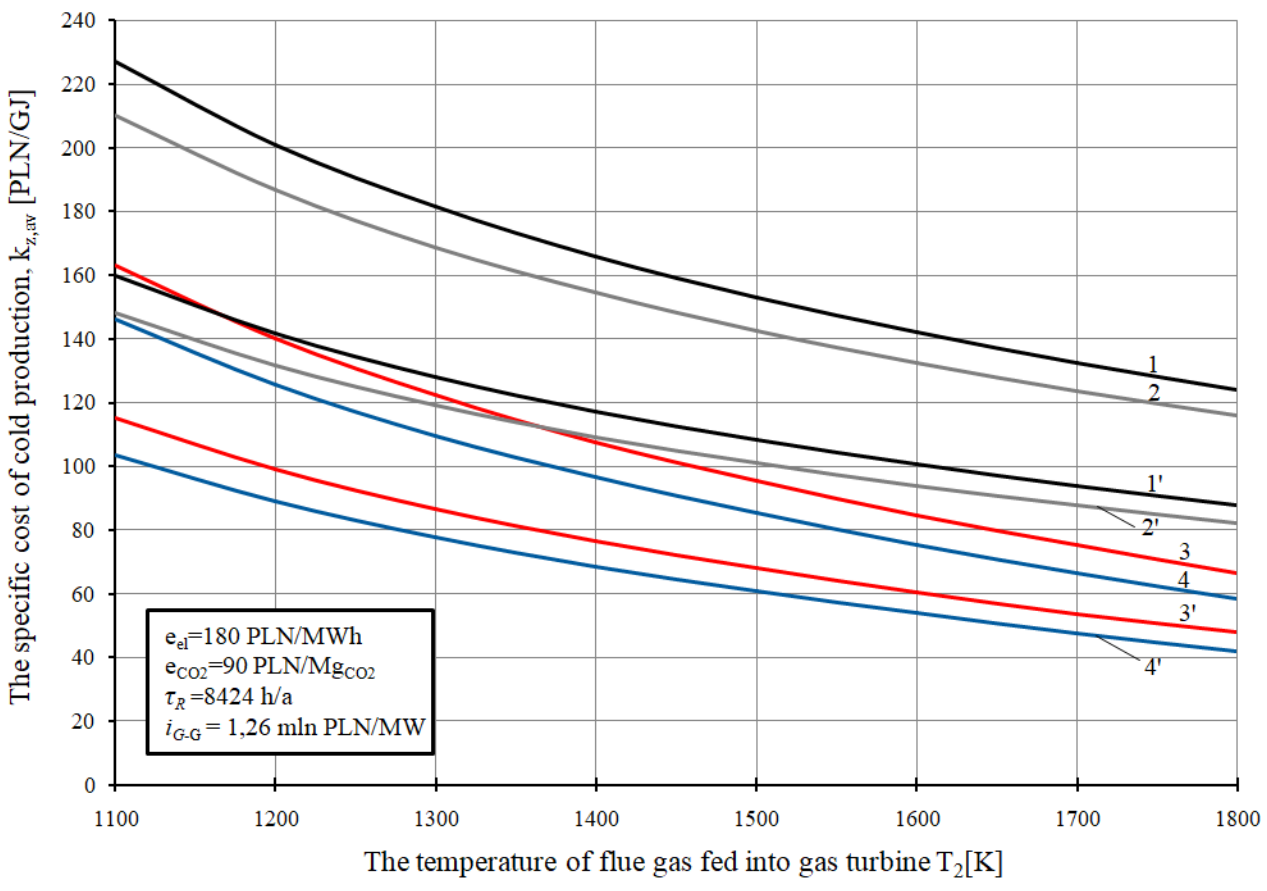

Figure 16. Values of specific cost of production of cold $k_{z, a v}$ in the gas-gas system comprising an absorption chiller and a turboexpander without regenerative heat exchanger in the function of the temperature of flue gas fed into gas turbine $T_{2}$. 


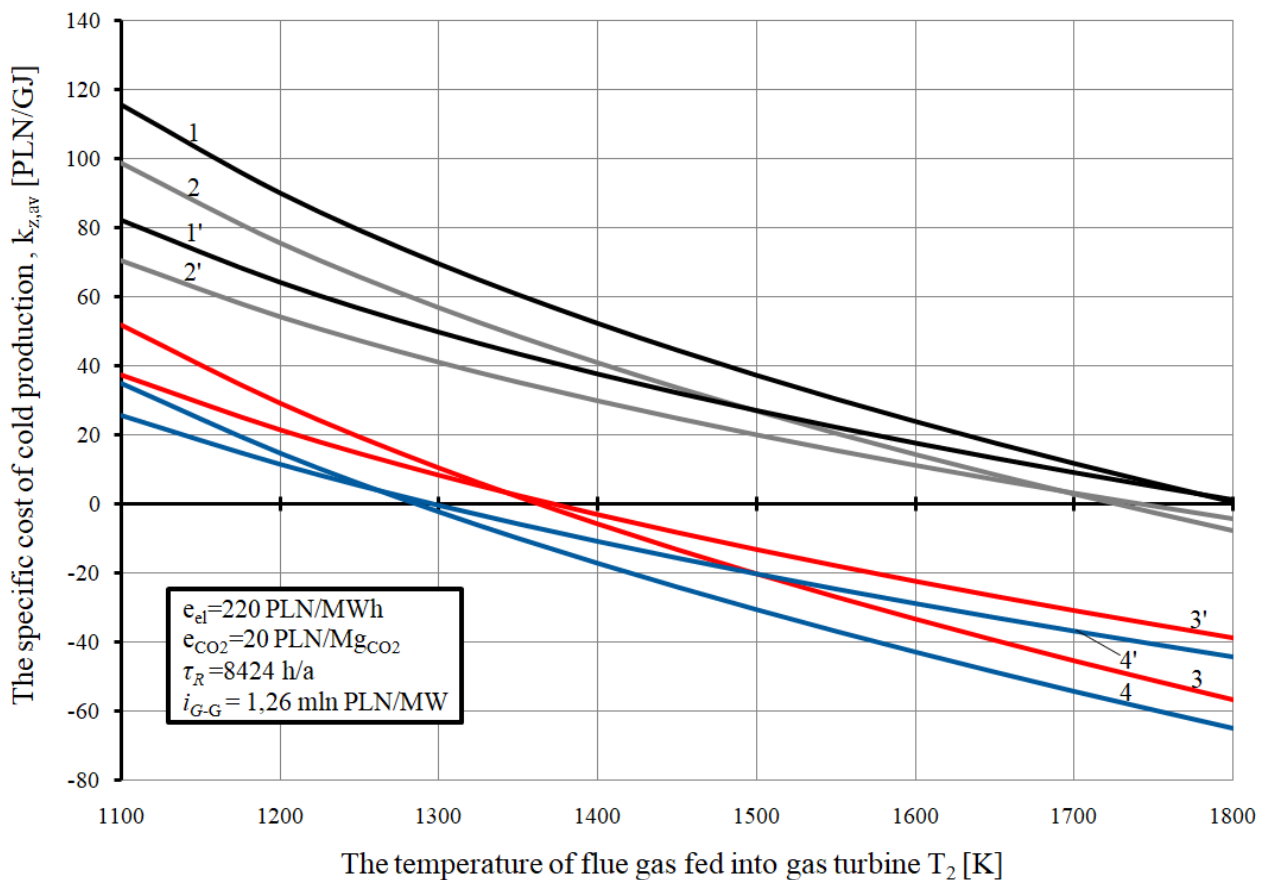

Figure 17. Values of specific cost of production of cold $k_{z, a v}$ in the gas-gas system comprising an absorption chiller and turboexpander excluding regenerative heat exchanger in the function of the temperature of flue gas fed into gas turbine $T_{2}$.

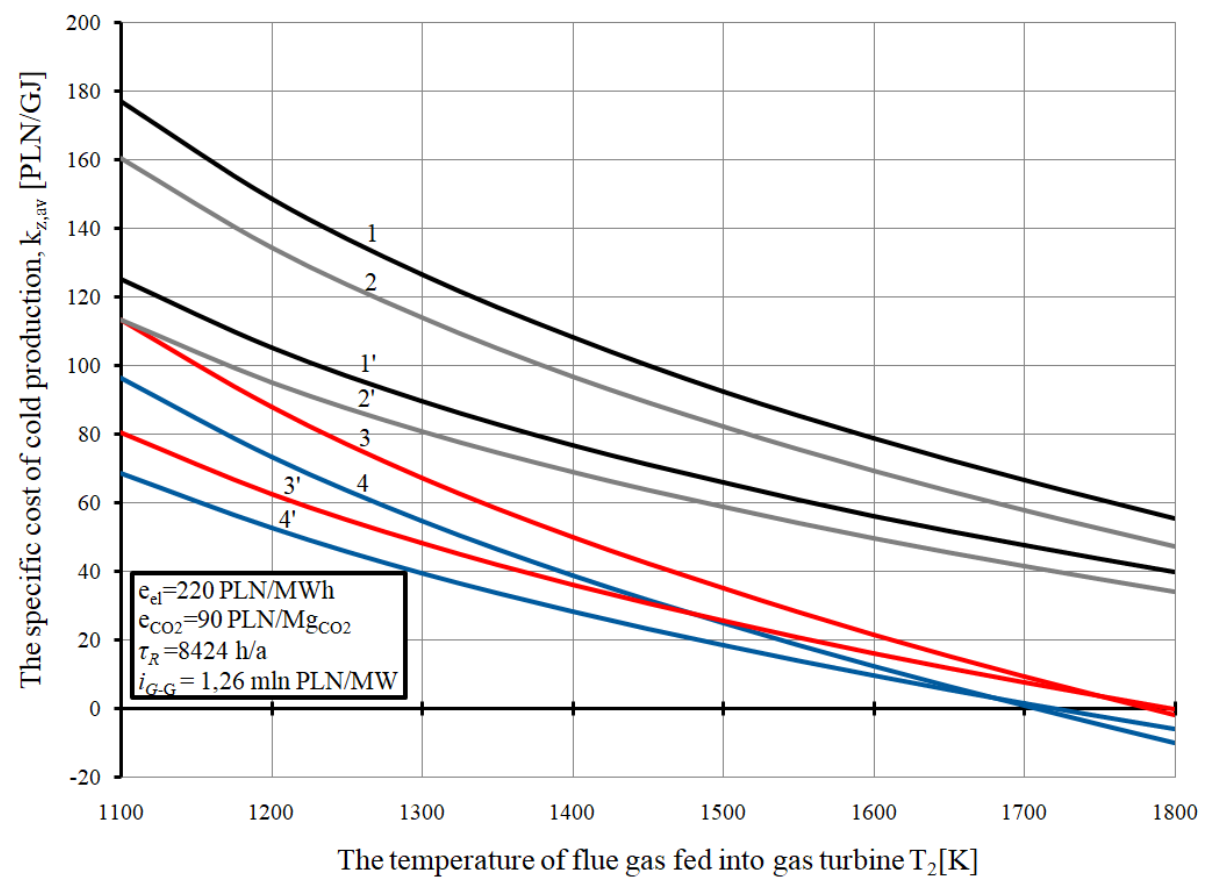

Figure 18. Values of specific cost of production of cold $k_{z, a v}$ in the gas-gas system comprising a turboexpander and absorption chiller excluding regenerative heat exchanger in the function of the temperature of flue gas fed into gas turbine $T_{2}$. 


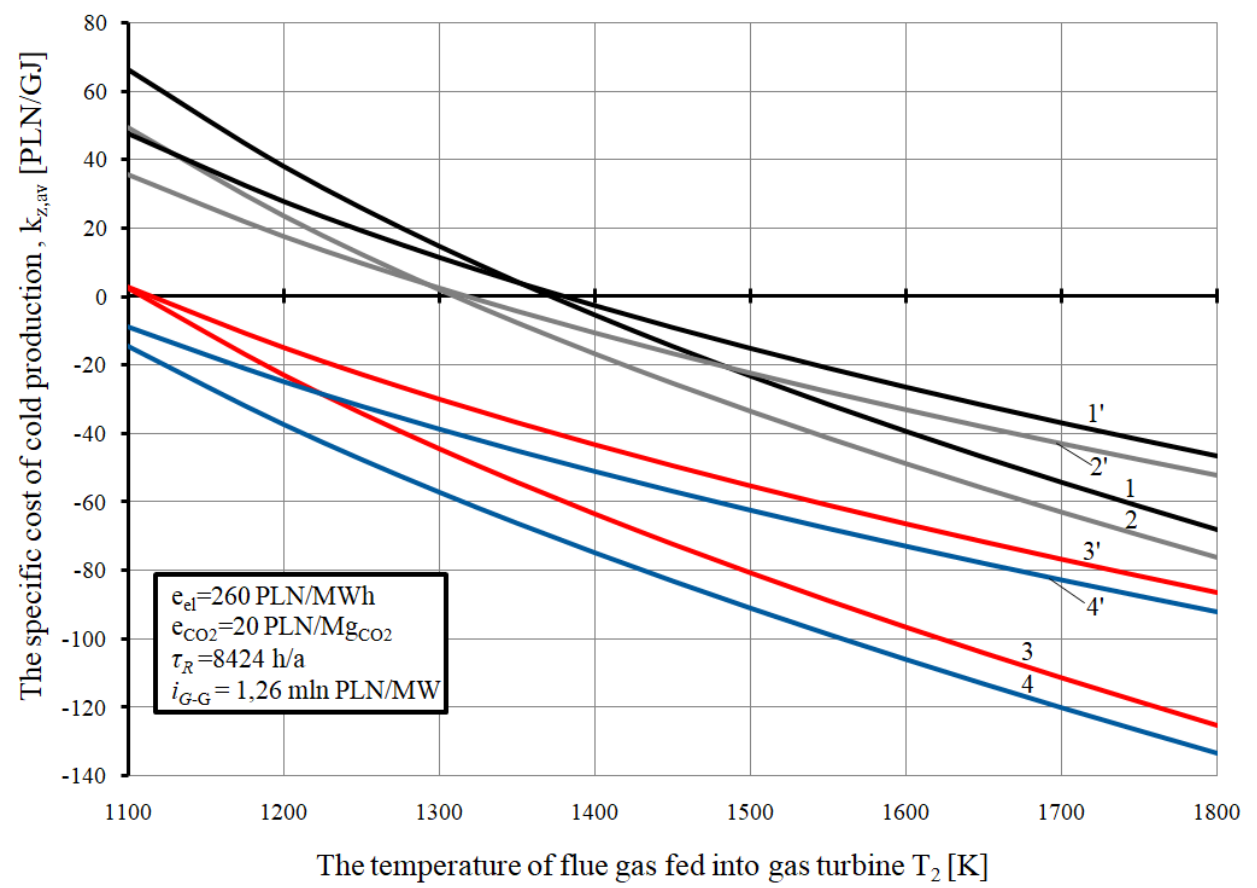

Figure 19. Values of specific cost of production of cold $k_{z, a v}$ in the gas-gas system comprising a turboexpander and absorption chiller excluding regenerative heat exchanger in the function of the temperature of flue gas fed into gas turbine $T_{2}$.

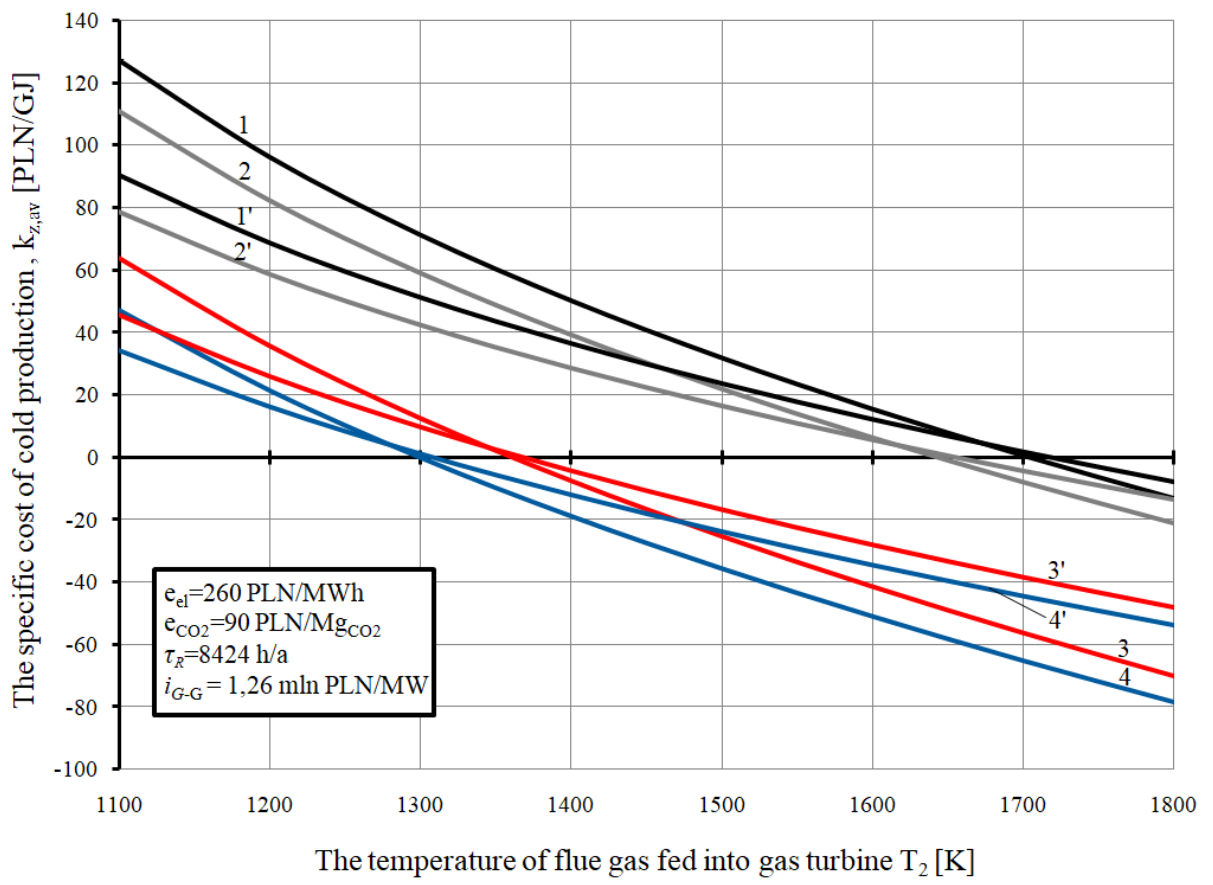

Figure 20. Values of specific cost of production of cold $k_{z, a v}$ in the gas-gas system comprising an absorption chiller and turboexpander excluding regenerative heat exchanger in the function of the temperature of flue gas fed into gas turbine $T_{2}$. 
Table 3. Summary of input data.

\begin{tabular}{ccccccccc}
\hline Symbols & $\mathbf{1}$ & $\mathbf{1}^{\prime}$ & $\mathbf{2}$ & $\mathbf{2}^{\prime}$ & $\mathbf{3}$ & $\mathbf{3}^{\prime}$ & $\mathbf{4}$ & $\mathbf{4}^{\prime}$ \\
\hline $\mathrm{e}_{\mathrm{c}}, \mathrm{PLN} / \mathrm{GJ}$ & 50 & 50 & 70 & 70 & 50 & 50 & 70 & 70 \\
$\mathrm{e}_{\mathrm{fuel}}, \mathrm{PLN} / \mathrm{GJ}$ & 24 & 24 & 24 & 24 & 20 & 20 & 20 & 20 \\
$i_{z}, \mathrm{PLN} / \mathrm{MW}$ & $0.5 i_{G-G}$ & $0.7 i_{G-G}$ & $0.5 i_{G-G}$ & $0.7 i_{G-G}$ & $0.5 i_{G-G}$ & $0.7 i_{G-G}$ & $0.5 i_{G-G}$ & $0.7 i_{G-G}$ \\
$\tau_{z}, \mathrm{~h} / \mathrm{a}$ & $0.7 \tau_{R}$ & $\tau_{z}=\tau_{R}$ & $0.7 \tau_{R}$ & $\tau_{z}=\tau_{R}$ & $0.7 \tau_{R}$ & $\tau_{z}=\tau_{R}$ & $0.7 \tau_{R}$ & $\tau_{z}=\tau_{R}$ \\
\hline
\end{tabular}

As we can see from the results of calculations presented in Figures 15-20, the specific cost of production of cold depends to the greatest extent on the price of electricity. We should note that when the specific cost associated with production of cold is compared in the system with the compressor chiller for the value $x=0.1$, i.e., for the same production of cold in both systems, the specific cost is lower in the compressor chiller.

When we calculate the cost in the system including a regenerative heat exchanger in the turboexpander in Figure 4, and under the assumption that the investment in the system comprising regeneration is the same as for the system without regeneration, it can be concluded that this cost decreases to a negligible extent and does not exceed 1 PLN/GJ in the entire range of variations in the temperature $T_{2}$, which is apparent in the system comprising a compressor chiller.

In order to achieve an increases cooling power of the absorption chiller (Formula (28)), the range of the temperatures of the flue gas $\Delta T=T_{8}-395$ applied to drive this chiller should be increased. The temperature span in this range, as already noted above, is relatively small and depends on the temperature of the gas fed into the gas turbine, as it ranges from around 63 to around $130 \mathrm{~K}$. To increase this range, it would be necessary to supplementary firing gas in the turboexpander in the channel which routes air from the preheater $\mathrm{N}$ to the turboexpander TE (this is known from the literature on gas-steam systems as the concept of so-called supplementary firing [2]), Figure 3.

As a result, the temperature $T_{6}$ and, consequently, the temperature $T_{8}$ will be increased. We should note, however, that combustion of fuel using burners located in the firing chamber located in the turboexpander channel N-TE leads to the conversion of a hierarchical engine-Figures 1-4-into a quasi-hierarchical engine, i.e., a dual-fuel engine. In general, the energy efficiencies of quasi-hierarchical systems are lower compared to those in hierarchical systems.

As a result of increasing the value $T_{6}$ by $\Delta T_{6}=100 \mathrm{~K}$ as a result of the combustion of gas in the channel N-TE, the temperature $T_{8}$ increases to $T_{8}^{\text {sup }}$. Depending on the temperature $T_{2} \in\langle 1100 ; 1800 \mathrm{~K}\rangle$, the temperature $T_{8}$ increases from the value $T_{8} \in\langle 458 ; 524 \mathrm{~K}\rangle$ to the value of $T_{8}^{\text {sup }} \in\langle 487 ; 555 \mathrm{~K}\rangle$. As a consequence of increasing $T_{6}$ by $\Delta T_{6}=200 \mathrm{~K}$, this temperature assumes the value of $T_{8}^{\text {sup }} \in$ $\langle 517 ; 586 \mathrm{~K}\rangle$. The ratio of the cooling power to the electrical capacity of the system in this case increases without gas combustion from the value $\dot{Q}_{z} / N_{e l}^{G-G}=\langle 0.32 ; 0.23\rangle$, Figure 11 , to $\dot{Q}_{z}^{\text {sup }} / N_{e l}^{G-G}=\langle 0.38 ; 0.25\rangle$, accompanied by the increase of the temperature $T_{6}$ by the value of $\Delta T_{6}=100 \mathrm{~K}$ and to the value $\dot{Q}_{z}^{\text {sup }} / N_{e l}^{G-G}=\langle 0.43 ; 0.26\rangle$ and is accompanied by the increase of the temperature $T_{6}$ by the value $\Delta T_{6}$ $=200 \mathrm{~K}$.

As a result of the increase of temperature $T_{6}$ by the value equal to $\Delta T_{6}$, we have to do with an increase of the efficiency $\eta_{T E}^{\max }$ to $\eta_{T E}^{\max \text { sup }}$. The values of these efficiencies are derived from the formula (50) after the value of zero substitutes $\eta_{R}$ and temperatures $T_{6}$ and $T_{6}^{\text {sup }}$ are used in the place of the temperature $T_{6 R}$. The values of $\eta_{T E}^{\max }\left(\eta_{T E}^{\max }\right.$ is derived for the value of $\left.\Delta T_{6}=0\right)$ and $\eta_{T E}^{\max \text { sup }}$ are presented in Figure 22.

The combustion of extra fuel in the turboexpander changes the efficiency of the generation of electricity in it. It is derived from the energy balance - Figure 21: 


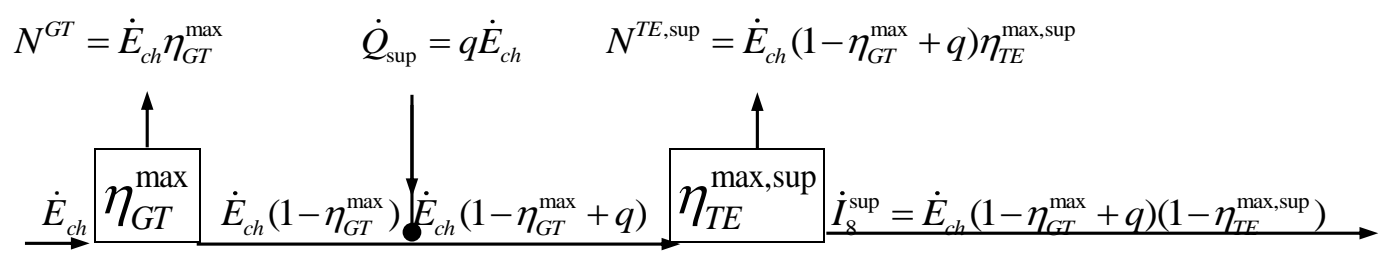

Figure 21. Energy balance of a hierarchical gas-gas engine with supplementary firing.

where:

$\dot{I}_{8}^{\text {sup }}$-enthalpy of flue gas fed into the chiller following supplementary firing of the fuel, $q$-level of supplementary firing in the engine, $q=\dot{Q}_{\text {sup }} / \dot{E}_{c h}$, on the basis of which we can derive the formula that represents the efficiency of electricity generation in a gas-gas engine in a system with supplementary firing:

$$
\eta_{G-G}^{\text {sup }}=\frac{N^{G T}+N^{T E, \text { sup }}}{\dot{E}_{c h}+\dot{Q}_{\text {sup }}}=\frac{\eta_{G T}^{\max }+\eta_{T E}^{\max , \text { sup }}-\eta_{G T}^{\max } \eta_{T E}^{\max \text { sup }}+q \eta_{T E}^{\max , \text { sup }}}{1+q}
$$

and if we assume that $q=0$, the equation in (30) represents the efficiency of the gas-gas engine without supplementary firing:

$$
\eta_{G-G}=\eta_{G T}^{\max }+\eta_{T E}^{\max }-\eta_{G T}^{\max } \eta_{T E}^{\max }
$$

The values of the efficiencies $\eta_{G-G}$ and $\eta_{G-G}^{\text {sup }}$ in the function of the temperatures of the flue gas fed into the gas turbine $T_{2}$ and value of $\Delta T_{6}$ are presented in Figure 22.

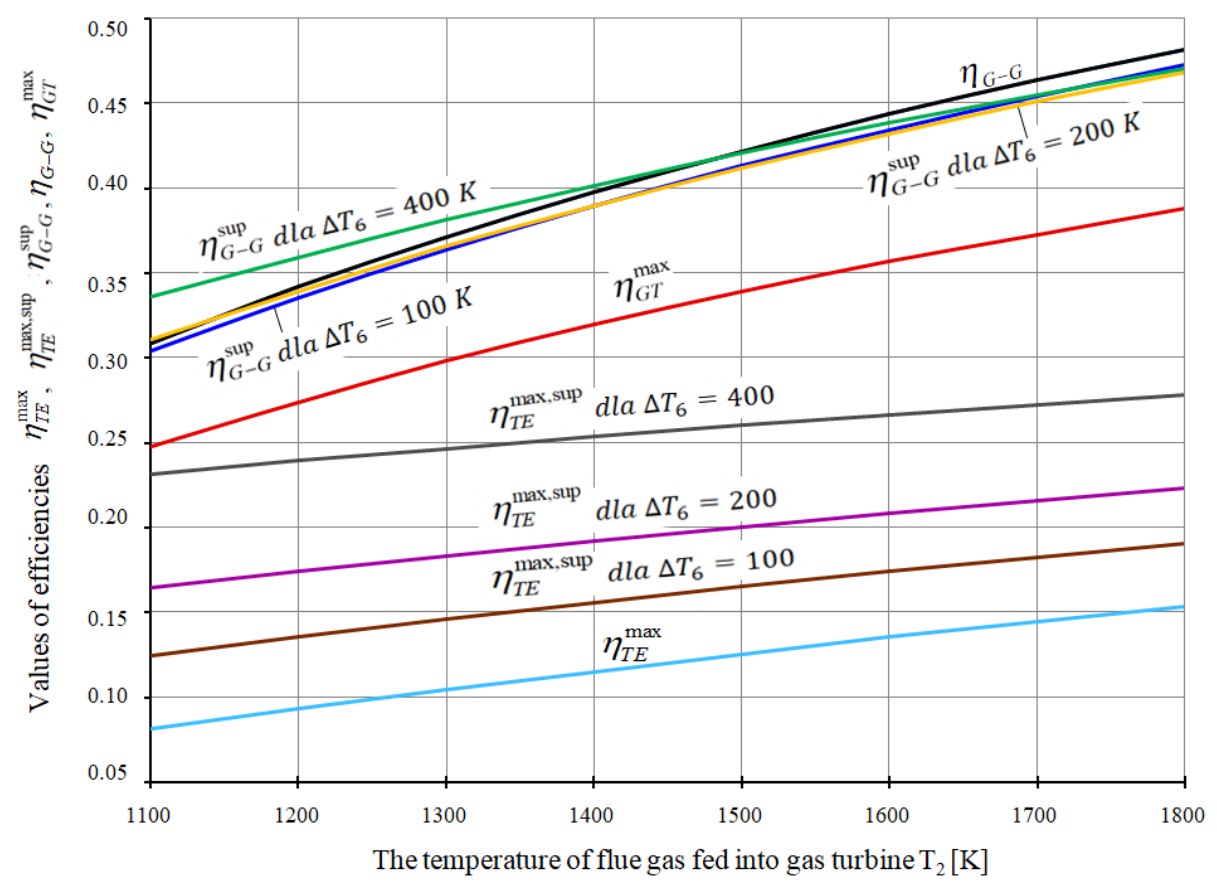

Figure 22. Values of efficiencies $\eta_{T E}^{\max }, \eta_{T E}^{\max , \text { sup }}, \eta_{G T}^{\max }, \eta_{G-G^{\prime}} \eta_{G-G}^{\text {sup }}$ in the function of the temperature $T_{2}$ of gas fed into gas turbine corresponding to temperature increase steps $\Delta T_{6}=T_{6}^{\text {sup }}-T_{6}$ equal to $\Delta T_{6}=0,100,200,400 \mathrm{~K}$.

As a result of dividing the relations (37) by (38), we derive the following:

$$
\frac{\eta_{G-G}^{\sup }}{\eta_{G-G}}=\frac{1}{1+q} \frac{1-\eta_{T E}^{\max , \text { sup }}+\frac{\eta_{T E}^{\max , d o p}}{\eta_{G T}^{\max }}(1+q)}{1+\frac{\eta_{T E}^{\max }}{\eta_{G T}^{\max }}-\eta_{T E}^{\max }}
$$


The stream of the chemical energy associated with extra fuel combustion is derived from the equation:

$$
\dot{Q}_{\text {sup }}=\dot{P}_{\text {sup }} W_{d}=\dot{C}_{T E}\left(T_{6}^{\text {sup }}-T_{6}\right)
$$

where: $\dot{P}_{\text {sup }}$-mass stream of supplementary fired gas, $W_{d}$ - gross calorific value of gas, where the stream corresponding to the heat capacity of flue gas $\dot{C}_{T E}$ routed into turboexpander is equal to approximately to heat capacity of air prior to supplementary firing, which in turn results from the energy balance in the air preheater N [1] and it equal to the stream of heat capacity of flue gas $\dot{C}_{G T}$ extracted from the gas turbine. The stream of heat capacity of flue gas $\dot{C}_{T G}$ results from the energy balance of the turbine, Figure 21:

$$
\dot{E}_{c h}-N^{G T}=\dot{E}_{c h}\left(1-\eta_{G T}^{\max }\right)=\dot{I}_{3}=\dot{C}_{G T}\left(T_{3}-T_{a m b}\right)
$$

where: $\dot{I}_{3}$-flux of enthalpy of the flue gas at the exhaust of the gas turbine.

By application of Equations (40) and (41) and on the basis of the relation that $\dot{C}_{T E} \approx \dot{C}_{G T}$, we can derive the level of supplementary firing:

$$
q=\frac{\dot{Q}_{\text {sup }}}{\dot{E}_{c h}}=\left(1-\eta_{G T}^{\max }\right) \frac{T_{6}^{\text {sup }}-T_{6}}{T_{3}-T_{a m b}} .
$$

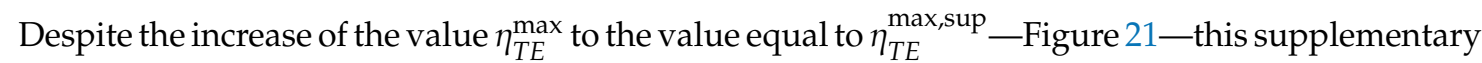
firing leads to the decrease of the efficiency of electricity generation in the engine. This is due to the fact that the ratio $\eta_{G-G}^{\text {sup }} / \eta_{G-G}$ is only slightly lower from one, since $\eta_{G-G}^{\text {sup }}$ is marginally smaller than $\eta_{G-G}$-Figure 22. We can note at this point that the revenue from the sale of electricity has a decisive effect on the economic profitability of the trigeneration system. Therefore, the additional gas combustion in the N-TE channel is not only unfeasible from the thermodynamic perspective, but in addition, due to the small increase in the cooling power of the system and the increase in investment associated with the supplementary firing and supplementary firing chamber in the N-TE channel, this option is economically unprofitable. The condition of profitability could then be a relatively high cold price and a low gas price. In such a case, only for the relatively large values of the temperature $T_{6}^{\text {sup }}$, i.e., for the high values of $\Delta T_{6}$, accompanied by the conditions when the temperatures $T_{6}^{\text {sup }}$ assume values in the range of $1100 \div 1400 \mathrm{~K}$ that are equal to the values of the temperatures $T_{2}$ of the flue gas fed into several year-old designs of gas turbines characterized by low efficiencies $\eta_{G T}$, the ratio $\eta_{G-G}^{\text {sup }} / \eta_{G-G}$ is only higher than one. The low efficiency of old designs of gas turbines results from the low heat resistance of the turbine blades, which leads to low values of permissible temperatures. Current designs include temperatures in the range of even above $1800 \mathrm{~K}$. However, the considerable role taken on by afterburning in the turboexpander means that the hierarchical turboexpander engine does not make any sense in terms of its technical aspects. In such a case, the turboexpander then takes on the function of a gas turbine.

The same characteristics of the courses as the ones that are contained in Figure 22 are assumed by the curves $\eta_{T E, R}^{\max }, \eta_{T E, R}^{\max , \text { sup }}$ for the engine including heat regeneration in the turboexpander-Figure 4-for the alternative when gas afterburning is applied in the N-TE channel in it. 


\section{Application of Turboexpander with Heat Regeneration in the Trigeneration System}

By performing the energy balance of the Joule's circulation of the turboexpander operating jointly with the regenerative heat exchanger $R$-Figures 4 and 23-we can obtain the formula representing its energy efficiency:

$$
\eta_{T E}=\frac{N_{T E, R}}{\dot{Q}_{\text {sup }}}=\frac{N_{i \exp }-N_{i C}}{\dot{Q}_{\text {sup }}}=\frac{\eta_{m}\left(T_{6 R}-T_{7 R}\right)-\frac{1}{\eta_{m}}\left(T_{5 R}-T_{a m b}\right)}{T_{6 R}-T_{N R}} .
$$

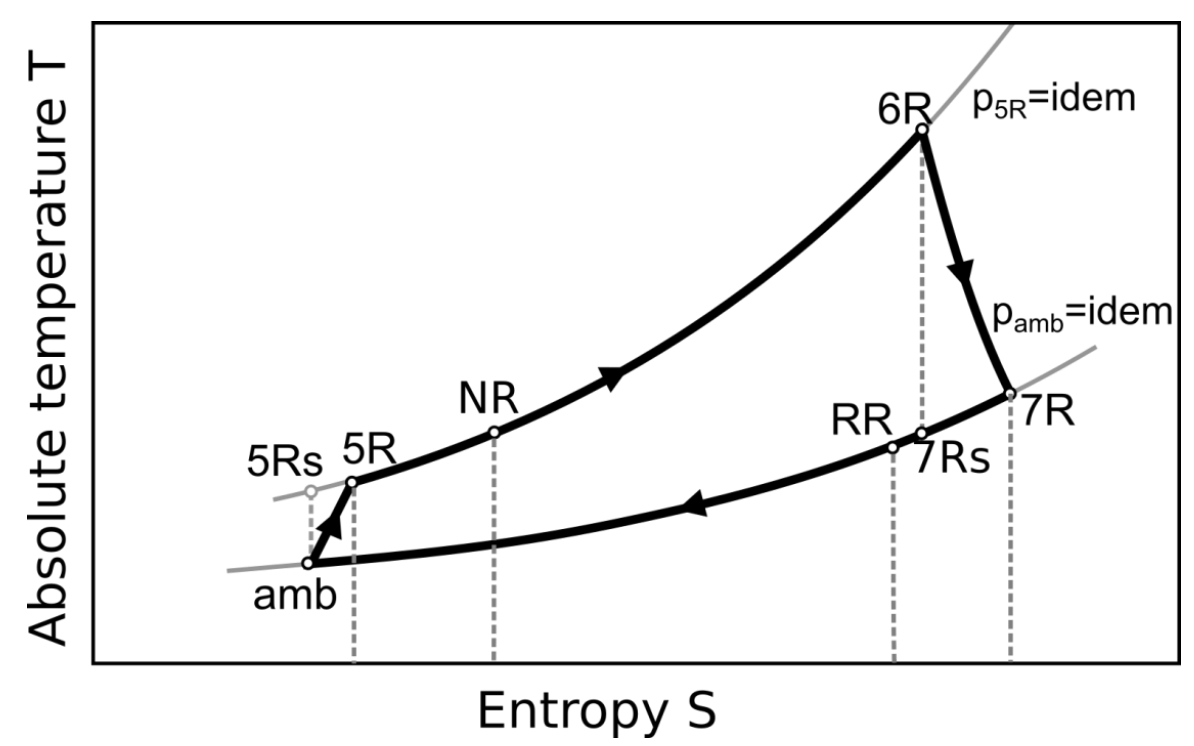

Figure 23. Joule's circulation in a turboexpander with heat regeneration (regeneration heat is equal to the area of the field below the RR-7R isobar and the entropy axis; the area is equal to the field below the section of the 5R-NR isobar (obvious, the relations: $T_{7 R}>T_{N R}, T_{R R}>T_{5 R}$ apply here as well).

After substituting the temperature of the circulating medium into Formula (43):

$$
\begin{gathered}
T_{5 R}=T_{a m b}+\frac{1}{\eta_{i}^{C}}\left(T_{5 R s}-T_{a m b}\right) \\
T_{7 R}=T_{6 R}-\eta_{i}^{T E}\left(T_{6 R}-T_{7 R s}\right) \\
T_{N R}=\left(1-\eta_{R}\right) T_{5 R}+\eta_{R} T_{7 R} \\
T_{5 R s}=T_{a m b} z_{T E, R} \\
T_{7 R s}=T_{6 R} / z_{T E, R}
\end{gathered}
$$

where:

$$
z_{T E, R}=\left(\frac{p_{5 R}}{p_{a m b}}\right)^{\frac{\kappa-1}{\kappa}}=\left(\frac{p_{6 R}}{p_{7 R}}\right)^{\frac{\kappa-1}{\kappa}} .
$$

we can obtain the final form of this formula (for the value of $z_{T E, R}^{\text {opt }}$ derived from Formula (53)):

$$
\eta_{T E, R}^{\max }=\frac{N_{T E, R}^{\max }}{\dot{Q}_{\text {sup }}}=\frac{\eta_{m} \eta_{i}^{T E} T_{6 R}\left(1-\frac{1}{z_{T E, R}^{o p t}}\right)-\frac{1}{\eta_{m} \eta_{i}^{C}} T_{a m b}\left(z_{T E, R}^{o p t}-1\right)}{T_{6 R}-\left(1-\eta_{R}\right) T_{a m b}\left[1+\frac{1}{\eta_{i}^{C}}\left(z_{T E, R}^{o p t}-1\right)\right]-\eta_{R} T_{6 R}\left[1-\eta_{i}^{T E}\left(1-\frac{1}{z_{T E, R}}\right)\right]}
$$


By substituting the value equal to zero $\eta_{R}=0$ in the place of $\eta_{R}$ in formula (43), we can obtain the formula representing the energy efficiency of the Joule circulation of the turboexpander excluding heat regeneration (Formula (8)).

The temperature of the air at the inlet to the turboexpander $T_{6 R}$ can be determined for the known value $T_{3}$ (Formula (18)) and an adopted value of $\Delta T_{1}$ :

$$
T_{6 R}=T_{3}-\Delta T_{1}
$$

The efficiency $\eta_{R}$ of the heat exchanger $R$, Figure 2, is defined by the relation:

$$
\eta_{R}=\frac{T_{N R}-T_{5 R}}{T_{7 R}-T_{5 R}}=\frac{T_{7 R}-T_{R R}}{T_{7 R}-T_{5 R}}
$$

where: $T_{N R}$-air temperature before the air pre-heater, $T_{R R}$-temperature of exhaust air fed from the regenerative heat exchanger into the stack, $T_{5 R}$-air temperature behind the compressor, $T_{7 R}$ - temperature of the air at the exhaust from the turboexpander.

The optimum value of the ratio of the pressure $z_{T E, R}^{\text {opt }}$ can be obtained on the basis of the equation below (we can derive it by adopting the condition that $d \eta_{T E} / d z_{T E, R}=0$ ):

$$
[(a+b) c-a d]\left(z_{T E, R}^{o p t}\right)^{2}+2(a e-b c) z_{T E, R}^{o p t}-(a+b) e+d b=0
$$

where:

$$
\begin{aligned}
& a=\frac{T_{a m b}}{\eta_{m} \eta_{i}^{C}}, b=\eta_{m} \eta_{i}^{T E} T_{6 R}, c=\frac{1-\eta_{R}}{\eta_{i}^{C}} T_{a m b}, \\
& d=T_{6 R}+\left(1-\eta_{R}\right) T_{a m b}\left(\frac{1}{\eta_{i}^{C}}-1\right)-\eta_{R} T_{6 R}\left(1-\eta_{i}^{T E}\right)
\end{aligned}
$$

$e=\eta_{R} \eta_{i}^{T E} T_{6 R}$

The negative root of the Equation (53) does not make any physical sense.

When we know the value $z_{T E, R}^{o p t}$ derived from the equation:

$$
z_{T E, R}^{o p t}=\frac{T_{5 R s}}{T_{a m b}}=\frac{T_{6 R}}{T_{7 R s}}=\left(\frac{p_{5 R}}{p_{a m b}}\right)_{o p t}^{\frac{\kappa-1}{\kappa}}
$$

we can determine the value $p_{5 R}^{\text {opt }}$, and by means of irreversible adiabates, and we can calculate the air temperatures behind the compressor $T_{5 R}$ and behind the turboexpander $T_{7 R}$ :

$$
\begin{aligned}
& T_{5 R}=T_{a m b}\left[1+\frac{1}{\eta_{i}^{C}}\left(z_{T E, R}^{o p t}-1\right)\right], \\
& T_{7 R}=T_{6 R}\left[1-\eta_{i}^{G T}\left(1-\frac{1}{z_{T E, R}^{\text {opt }}}\right)\right] .
\end{aligned}
$$

If we have the temperatures $T_{5 R}$ and $T_{7 R}$ as input, we can derive the temperatures $T_{N R}, T_{R R}$ from the equations (52).

Figures 24-26 contains the results of thermodynamic calculations for a gas-gas system working with a turboexpander with a regenerative heat exchanger-Figures 4 and 21-and for comparison purposes, for a system without regeneration [1]. 


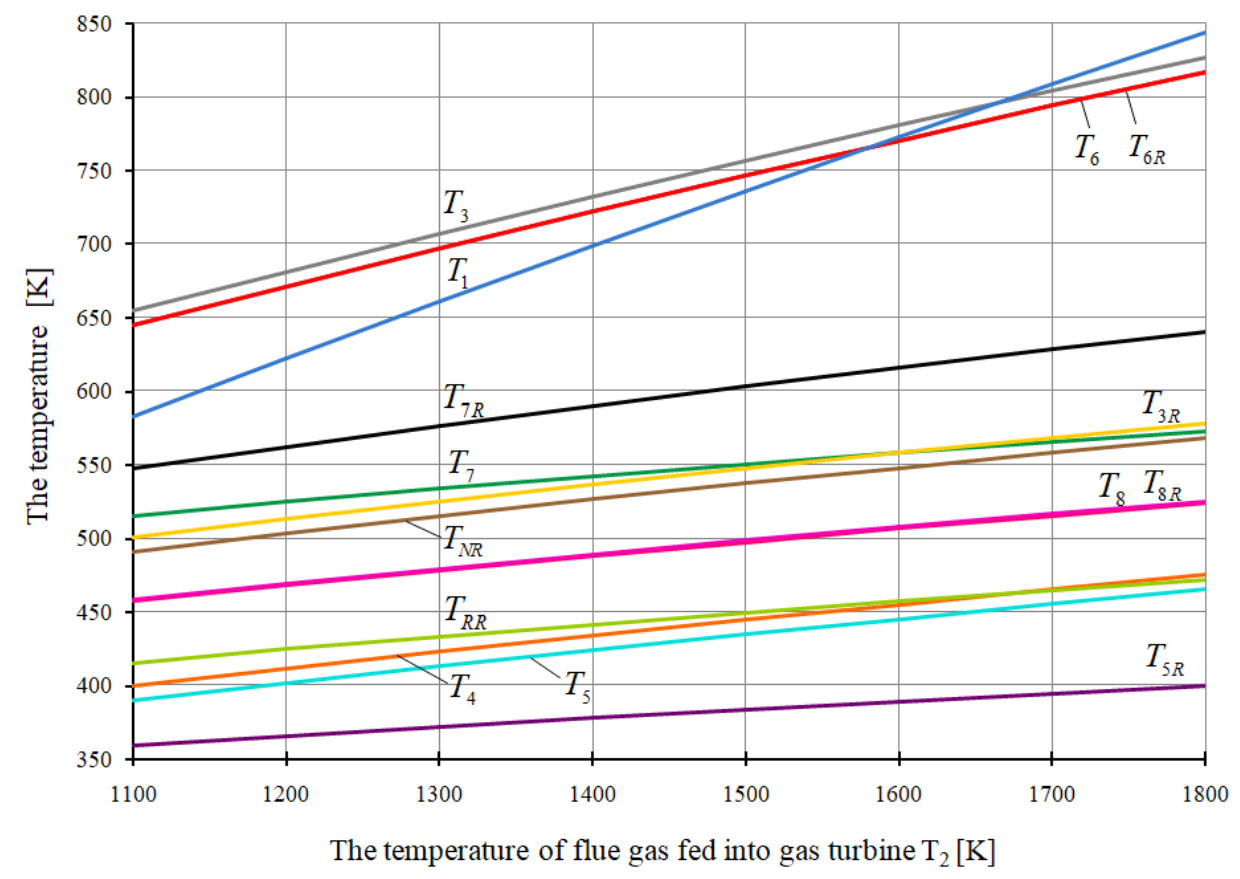

Figure 24. Temperatures $T_{1}, T_{3}, T_{4}, T_{5}, T_{6}, T_{7}, T_{8}$ and $T_{N R}, T_{R R}, T_{3 R}, T_{5 R}, T_{6 R}, T_{7 R}, T_{8 R}$ in the function of the temperatures of the flue gas fed into the gas turbine $T_{2}$.

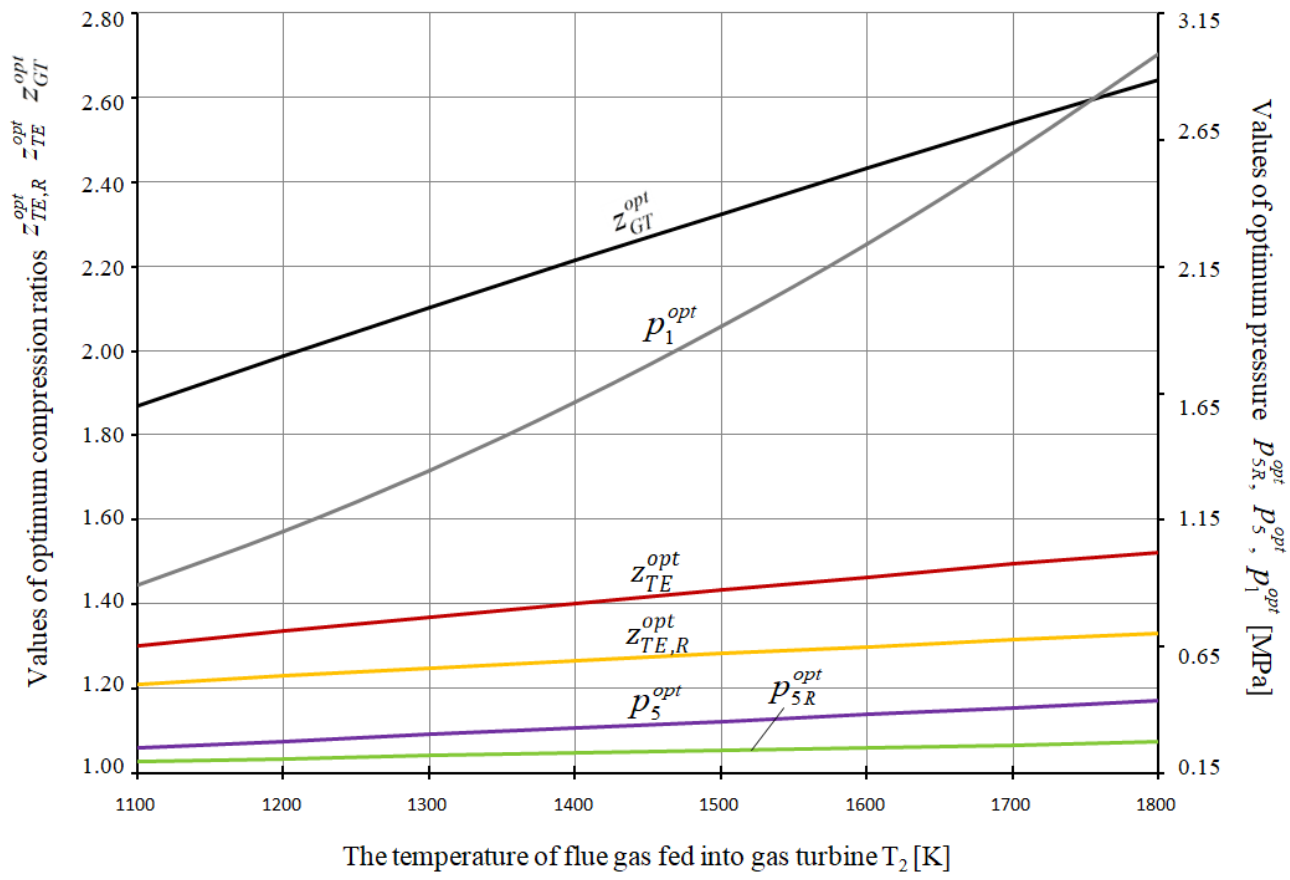

Figure 25. Values of optimal pressure $p_{1}^{o p t}, p_{5}^{o p t}, p_{5 R}^{o p t}$ and optimum compression ratios $z_{T E}^{o p t}, z_{T E, R^{\prime}}^{o p t} z_{T G}^{o p t}$ in the function of the temperatures of the flue gas fed into the gas turbine $T_{2}$. 


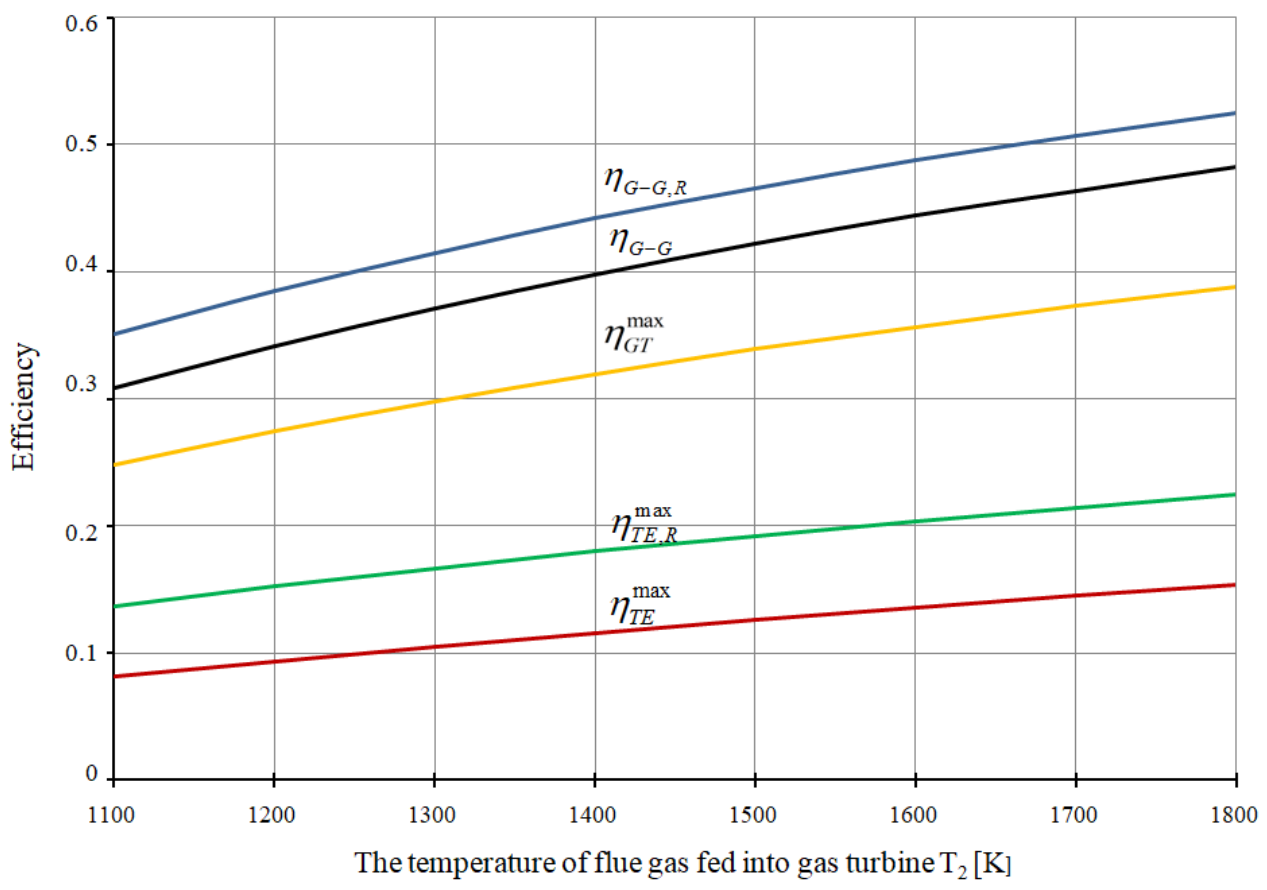

Figure 26. Values of efficiency $\eta_{G-G}, \eta_{G-G, R^{\prime}} \eta_{T E}^{\max }, \eta_{T E, R^{\prime}}^{\max } \eta_{G T}^{\max }$ in the function of the temperatures of the flue gas fed into the gas turbine $T_{2}$.

As we can see from the results of calculations presented in Figures 21-26, the application of heat regeneration in the turboexpander, Figure 4, is feasible from the thermodynamic perspective. This is due to the fact that this leads to the increase of the efficiency $\eta_{T E, R}^{\max }$ accompanied by $\eta_{G-G, R^{\prime}}$, hence, the power of the turboexpander increases as well, coupled with the electrical power of the entire system. In such a case, the ratio of the power of the turboexpander to the power of the gas turbine is equal to around $N_{R}^{T E} / N^{G T} \approx 15,5 \%$, whereas in a system excluding regeneration-Figures 1-3-this ratio is equal to around $N^{T E} / N^{G T} \approx 15 \%$ [1]. As a result, the temperature of the flue gas $T_{8 R}$ increases slightly, i.e., by about $1{ }^{\circ} \mathrm{C}$ above $T_{8}$ (these temperature overlap in Figure 24), which nevertheless slightly increases of the cooling power of the thermal absorption chiller (Formula (28)).

It is important to establish an answer to the question regarding the economic feasibility of the gas-gas system with a turboexpander with a regenerative heat exchanger side by side with the conclusion concerning its mere better parameters from a thermodynamic point of view. A necessary condition for this needs to involve an increase in the revenue gained from the sale of additional electricity generated in the turboexpander, as well as the cold production in the gas-gas system that needs to be not smaller than the increase in annual capital (depreciation and finance costs) and operating costs (maintenance and renovations) related to the increase of capital expenditure related to the development and building of a regenerative heat exchanger. Even on the assumption, as already noted above, that the increase in the necessary investment is zero, i.e., when the cost of the investment is identical as for the system excluding regenerative heat exchanger, the decrease in the specific cost of cold production is negligible, as it does not exceed the value of 1 PLN/ GJ. If the increase in this cost was accounted for in the calculations, the reduction in specific cost would be at best equal to zero. Therefore, the use of the regenerative heat exchanger does not make any economic sense. Therefore, it can be concluded that the design of a gas-gas system with a turboexpander operating for the purpose of heat regeneration, both in a system with a compressor and thermal absorption chiller, is completely economically unfeasible.

In addition, the application of regenerative heat exchanger in a gas turbine does not have any economic justification. The temperature of the gas extracted from the gas turbine regeneration exchanger is relatively low, i.e., around $100 \mathrm{~K}$ lower than the temperature of the gas extracted from 
the turbine operating without regenerative heat exchanger, Figures 1 and 4 [1]. Consequently, the air in the $N$ pre-heater, which drives the turboexpander, heated by these flue gases, results in a relatively low turboexpander power equal to only $5.5 \%-8 \%$ of the power of the gas turbine set (5.5\% and $8 \%$ correspond in turn to the $T_{2}$ flue gas temperatures inlet to gas turbine of 1100 and $1800 \mathrm{~K})$. Hence, the production of electricity in the turboexpander will not balance the capital costs incurred in its construction and the costs of its operation. The power ratio $N^{T E} / N^{G T}$ in the system in which heat regeneration would be used both in the gas turbine and in the turboexpander is almost identical. Therefore, the use of regeneration, which should be emphasized once again, has no economic justification.

\section{Conclusions and Final Remarks}

The article analyzes the specific costs of cold production in the compressor chiller. What is more, they were compared with the costs in an absorption chiller. The work is, therefore, highly innovative. Just as innovative as the subject of unit costs of cold production combined with the production of electricity and heat, bringing great practical knowledge about their amount and allowing investors to make rational investment decisions, the equally innovative is the hierarchical gas-gas system of this trigeneration production process.

Among the variety of trigeneration systems applied for combined production of electricity, heat and cold and analyzed in this paper, the system with a compressor chiller is more economically feasible. The specific cost of cold production in it-combined with the constant production as in the system with an absorption chiller-is lower than the cost of production in a system comprising an absorption chiller. The advantage of this system is associated with the fact that that the chiller is driven with electricity; hence, its refrigerating power can be arbitrarily large. On the other hand, in an absorption chiller, it is very much limited by the temperature range of the enthalpy of the flue gas from the engine and applied to feed it. In a system with a compressor chiller, heat production is also considerable, i.e., over 3 times higher compared to the system comprising an absorption chiller, and the revenue from its sales is formed by the cost of avoiding cold production. This is due to the use of the total range of the available temperature corresponding to the enthalpy of flue gases from the engine for heat production, whereas in the system with an absorption chiller it, for the same temperature range-Figures 1 and 3-this enthalpy also serves for the purposes of the production of cold.

We can also note that the production of electricity in systems with both a compressor and absorption chillers increases along with the higher efficiency of its production in the gas turbine set and in the turboexpander and, therefore, it increases with the higher temperatures $T_{2}$ of the flue gas extracted from the gas turbine set. In addition, the revenue from the sale of electricity is, similar to the revenue from the sale of heat, equal to the cost of avoided cold production. Thus, as the temperature increases, the specific cost of cold production decreases significantly. We should note, however, that the revenue from the sale of electricity in the system with a compressor chiller decreases as its refrigerating power increases. The compressor chiller is, as has already been pointed out, driven by electricity generated in the system.

It is also important to find the answer to the question: does the use of regenerative heat exchangers in a hierarchical gas-gas engine, both in the turboexpander and gas turbine cycle, increase the efficiency of electricity production in it and make it economically profitable? The answer is clearly no. Heat exchangers increase the investment in the engine, and thus increase capital costs (both its depreciation and cost of finance) and the costs of operating the engine (maintenance and repair), and at the same time the increase in electricity production is so small that the reduction in the specific cost of cold production as a consequence in the system with a heat chiller and compressor is zero in the best circumstances.

Finally, it should be stated explicitly that the great advantage of hierarchical gas-gas engines that is impossible to overestimate, both in terms of the production of only electricity and for cogeneration and trigeneration systems, is associated with the potential to build them in the areas where there is a lack of water. 
Author Contributions: Methodology, R.B., A.H.-S.; software, R.B.; formal analysis, A.H.-S.; resources, Z.B., W.S., A.O.; data curation, A.H.-S.; writing—original draft preparation, R.B., A.H.-S.; writing—review and editing, R.B., A.H.-S.; visualization, A.O., W.S.; supervision, R.B. All authors have read and agreed to the published version of the manuscript.

Funding: This research received no external funding.

Conflicts of Interest: The authors declare no conflict of interest.

\section{Nomenclature}

G-electric generator,

$\mathrm{K}$-combustion chamber of the gas turbine,

$\mathrm{N}$-air preheater;

$C_{T E}$-low-pressure compressor,

$C_{G T}$-high-pressure compressor of the gas turbine,

GT-turbine section of the gas turbine,

TE-turboexpander,

$\mathrm{HE}$-heat exchanger,

$\mathrm{Z}$-compressor or absorption chiller

$\mathrm{R}$-regenerative heat exchanger

$N^{G T}$-gas turbine power,

$N^{T E}$ _turboexpander power,

$\dot{Q}_{c}$-thermal power of the heat exchanger,

$\dot{Q}_{z}$-cooling power of either the compressor or absorption chiller,

$\dot{E}_{c h}$-the stream of the chemical energy of the gas burned in the gas turbine,

$\eta_{G T}$ - energy efficiency of a gas turbine,

$\eta_{T E}$ - energy efficiency of a turbo-expander,

$\kappa$-isentropic exponent of the circulating medium,

$a_{e l}, a_{c}, a_{z}, a_{f u e l}, a_{\mathrm{CO}_{2}}, a_{\mathrm{CO}}, a_{\mathrm{SO}_{2}}, a_{\mathrm{NO}_{X}}, a_{d u s t}, b_{\mathrm{CO}_{2}}$-terms of the exponents representing the changes in time of electricity, heat, cold, fuel prices, tariff charges on the environmental emissions, $\mathrm{CO}_{2}$ allowances;

$e_{e l}^{t=0}, e_{f u e l}^{t=0}, e_{c}^{t=0}, e_{z}^{t=0}, e_{\mathrm{CO}_{2}}^{t=0}, p_{\mathrm{CO}_{2}}^{t=0}$, etc.-initial prices of electricity, fuel, heat, cold, $\mathrm{CO}_{2}$ emission allowances, etc.,

$\dot{E}_{c h}$-stream of the chemical energy of fuel combustion in the gas turbine,

$J_{G-G}, J_{z}$-investment in the gas-gas system and chiller,

$P_{\mathrm{CO}_{2}}, P_{\mathrm{CO}}, P_{\mathrm{NO}}, P_{\mathrm{SO}_{2}}, p_{\text {dust }}$ - specific charges on $\mathrm{CO}_{2}, \mathrm{CO}, \mathrm{NO}_{x}, \mathrm{SO}_{2}$, particulate matter emissions,

$r$-interest rate of capital investment,

$x_{\text {sal }, \text { t,ins }}$-factor applied to account for the cost of remuneration, taxes, insurance, etc.,

$z$-discount coefficient (freezing coefficient) on investment $J$,

$\delta_{\text {serv }}$-annual rate of fixed cost relative to the investment,

$\varepsilon_{z}=\dot{Q}_{z} / N_{z}$-thermal efficiency of chiller,

$\varepsilon_{e l}$-internal electric load of the system,

$\eta_{G}$-efficiency of the electric generator,

$\rho_{\mathrm{CO}_{2}}, \rho_{\mathrm{CO}}, \rho_{\mathrm{NO}_{x}}, \rho_{\mathrm{SO}_{2}}, \rho_{\text {dust }}-\mathrm{CO}_{2}, \mathrm{CO}, \mathrm{NO}_{\mathrm{x}}, \mathrm{SO}_{2}$, particulate matter emissions per specific value of the chemical energy of fuel,

$\sigma_{R}=E_{e l, R} / Q_{R c}$-ratio of annual electricity production to annual heat production,

$\tau_{R}$-annual duration of the operation of the trigeneration system,

$\tau_{z}$-annual operating time of a chiller expressed in hours,

$i_{z}$-specific investment in the chiller,

$k_{z, a v}$-specific values of production of cold,

$\varepsilon_{z a b s}$ - thermal efficiency of cooling process of the absorption chiller,

$\dot{I}_{8}^{\text {sup }}$ —enthalpy of flue gas fed into the chiller following supplementary firing of the fuel,

$q$-level of supplementary firing in the engine,

$\dot{P}_{\text {sup }}$-mass stream of supplementary fired gas,

$W_{d}$-gross calorific value of gas,

$\dot{I}_{3}$-flux of enthalpy of the flue gas at the exhaust of the gas turbine. 


\section{References}

1. Bartnik, R.; Buryn, Z.; Hnydiuk-Stefan, A. Thermodynamic and economic analysis of a gas turbine set coupled with a turboexpander in a hierarchical gas-gas system. Energy 2020, 190, 116394. [CrossRef]

2. Gas Turbine World. 2007-2008 GTW Handbook; Pequot Publication, Inc.: Southport, UK, 2007; Volume 26.

3. Dadsetani, R.; Sheikhzadeh, G.A.; Safaei, M.R.; Alnaqi, A.A.; Amiriyoon, A. Exergoeconomic optimization of liquefying cycle for noble gas argon. Heat Mass. Transf. 2019, 55, 1995. [CrossRef]

4. Bartnik, R. Elektrownie i Elektrociepłownie Gazowo-Parowe; Efektywność Energetyczna i Ekonomiczna, WNT: Warszawa, Poland, 2009.

5. Mahsa, R.; Mehdi, M.; Zhao, H.; Zhen, H. Chemical looping technology in CHP (combined heat and power) and CCHP (combined cooling heating and power) systems: A critical review. Appl. Energy 2019, 253, 113544.

6. Mohammad, J.D.; Chang, K.Y. Modeling and extensive analysis of the energy and economics of cooling, heat, and power trigeneration (CCHP) from textile wastewater for industrial low-grade heat recovery. Energy Convers. Manag. 2020, 205, 112451.

7. Srinivas, G. Low-grade waste heat recovery for simultaneous chilled and hot water generation. Appl. Therm. Eng. 2012, 42, 191-198.

8. Yousef, N.; Dabwan, G. Pei: A novel integrated solar gas turbine trigeneration system for production of power, heat and cooling: Thermodynamic-economic-environmental analysis. Renew. Energy 2020, 152, 925-941.

9. Sztekler, K.; Kalawa, W.; Mika, L.; Krzywanski, J.; Grabowska, K.; Sosnowski, M.; Nowak, W.; Siwek, T.; Bieniek, A. Modeling of a Combined Cycle Gas Turbine Integrated with an Adsorption Chiller. Energies 2020, 13, 515. [CrossRef]

10. Valencia, O.G.; Acevedo, P.C.; Duarte, F.J. Thermo-Economic Assessment of a Gas Microturbine-Absorption Chiller Trigeneration System under Different Compressor Inlet Air Temperatures. Energies 2019, 12, 4643. [CrossRef]

11. Jamaluddin, K.; Wan Alwi, S.R.; Abdul Manan, Z.; Hamzah, K.; Klemeš, J.J. A Process Integration Method for Total Site Cooling, Heating and Power Optimisation with Trigeneration Systems. Energies 2019, 12, 1030. [CrossRef]

12. Zarzycki, R.; Kacprzak, A.; Bis, Z. The Use of Direct Carbon Fuel Cells in Compact Energy Systems for the Generation of Electricity, Heat and Cold. Energies 2018, 11, 3061. [CrossRef]

13. Conte, B.; Bruno, J.C.; Coronas, A. Optimal Cooling Load Sharing Strategies for Different Types of Absorption Chillers in Trigeneration Plants. Energies 2016, 9, 573. [CrossRef]

14. Underwood, C.; Ng, B.; Yik, F. Scheduling of Multiple Chillers in Trigeneration Plants. Energies 2015, 8, 11095-11119. [CrossRef]

15. Mohammad, M.S.; Mohammad, R.S.; Arturo, S.; Leon, U.K.; Marjan, G.R.M. Energetic Analysis of Different Configurations of Power Plants Connected to Liquid Chemical Looping Gasification. Processes 2019, 7, 763. [CrossRef]

16. Patel, B.; Desai, N.B.; Kachhwaha, S.S. Optimization of waste heat based organic Rankine cycle powered cascaded vapor compression-absorption refrigeration system. Energy Convers. Manag. 2017, 154, 576-590. [CrossRef]

17. Rashidi, H.; Khorshidi, J. Exergoeconomic analysis and optimization of a solar based multigeneration system using multiobjective differential evolution algorithm. J. Clean. Prod. 2018, 170, 978-990. [CrossRef]

18. Kody, K.M. Powell Thermodynamic and economic analysis of different cogeneration and trigeneration systems based on carbon dioxide vapor compression refrigeration systems. Appl. Therm. Eng. 2020, 164, 114503.

19. Abdi-khanghah, M.; Alrashed, A.A.A.A.; Hamoule, T.; Behbahani, R.M.; Goodarzi, M. Toluene methylation to para-xylene. J. Anal. Calorim. 2019, 135, 1723. [CrossRef]

20. Szargut, J. Termodynamika Techniczna; Wydawnictwo Politechniki Śląskiej: Gliwice, Poland, 1998.

21. Bartnik, R. The Modernization Potential of Gas Turbines in the Coal-Fired Power Industry. In Thermal and Economic Effectiveness; Springer: London, UK, 2013.

22. Bartnik, R.; Buryn, Z.; Hnydiuk-Stefan, A. Ekonomika Energetyki w Modelach Matematycznych z Czasem Ciagtym; Wydawnictwo Naukowe PWN: Warszawa, Poland, 2017. 
23. Bartnik, R.; Bartnik, B.; Hnydiuk-Stefan, A. Optimum Investment Strategy in the Power Industry. In Mathematical Models; Springer: New York, NY, USA, 2016.

24. Bartnik, R.; Buryn, Z.; Hnydiuk-Stefan, A. Investment Strategy in Heating and CHP. In Mathematical Models; Springer: London, UK, 2017.

(C) 2020 by the authors. Licensee MDPI, Basel, Switzerland. This article is an open access article distributed under the terms and conditions of the Creative Commons Attribution (CC BY) license (http://creativecommons.org/licenses/by/4.0/). 